\title{
A graphic approach to identities induced from multi-trace Einstein-Yang-Mills amplitudes
}

\author{
Yi-Jian $\mathbf{D u} u^{a, b, c}$ and Linghui $\mathbf{H o u}^{a}$ \\ ${ }^{a}$ Center for Theoretical Physics, School of Physics and Technology, Wuhan University, \\ No. 299 Bayi Road, Wuhan 430072, China \\ ${ }^{b}$ College of Science, Tibet University, \\ No. 10 Zangda East Road, Lasa, 850000, China \\ ${ }^{c}$ Suzhou Institute of Wuhan University, \\ No. 377 Linquan Street, Suzhou, 215123, China \\ E-mail: yijian.du@whu.edu.cn, hlh@whu.edu.cn
}

\begin{abstract}
Symmetries of Einstein-Yang-Mills (EYM) amplitudes, together with the recursive expansions, induce nontrivial identities for pure Yang-Mills amplitudes. In the previous work [1], we have already proven that the identities induced from tree level singletrace EYM amplitudes can be precisely expanded in terms of BCJ relations. In this paper, we extend the discussions to those identities induced from all tree level multi-trace EYM amplitudes. Particularly, we establish a refined graphic rule for multi-trace EYM amplitudes and then show that the induced identities can be fully decomposed in terms of BCJ relations.
\end{abstract}

KEYwords: Scattering Amplitudes, Gauge Symmetry

ARXIV EPRINT: 1910.04014 


\section{Contents}

1 Introduction 1

2 Refined graphic rule for multi-trace EYM amplitudes and the induced identities

2.1 Refined graphic rule

2.2 Examples for the refined graphic rule 8

$\begin{array}{lll}2.3 & \text { Induced identities by refined graphs } & 10\end{array}$

3 Identities induced from $A\left(1, \cdots, r \mid \mathbf{2} \| h_{1}\right)$ as combinations of BCJ rela$\begin{array}{ll}\text { tions } & 11\end{array}$

3.1 Example-1: the identity (2.19) induced from $A\left(1,2, \cdots, r \mid \mathbf{2} \| h_{1}\right) \quad 11$

3.2 Example-2: the identity (2.21) induced from $A\left(1,2, \cdots, r \mid \mathbf{2} \| h_{1}\right) \quad 15$

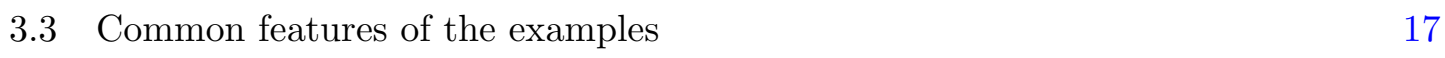

4 General study $\quad 19$

$\begin{array}{lll}4.1 \text { Skeletons and components } & 21\end{array}$

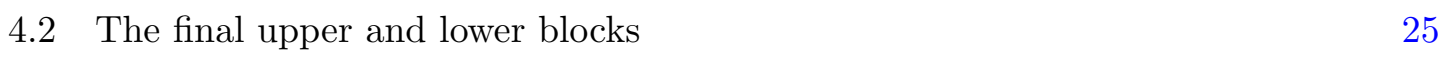

$\begin{array}{ll}4.3 \text { Physical and spurious graphs } & 26\end{array}$

4.4 Expanding induced identities in terms of BCJ relations 28

$\begin{array}{llr}5 & \text { Conclusions } & 29\end{array}$

$\begin{array}{ll}\text { A Recursive expansions and BCJ relations } & \mathbf{3 0}\end{array}$

A.1 Recursive expansions of multi-trace EYM amplitudes 30

A.2 Identities induced from multi-trace EYM amplitudes 32

$\begin{array}{lll}\text { A.3 BCJ relations } & 33\end{array}$

B Proof of eq. (3.5) $\quad 33$

$\begin{array}{ll}\text { C The sign in eq. (4.10) } & 36\end{array}$

\section{Introduction}

It has been proven that any tree level multi-trace Einstein-Yang-Mills (EYM) amplitude $A^{(m, s)}$ with $m$ gluon traces and $s$ gravitons can be recursively expanded in terms of the amplitudes $A^{\left(m^{\prime}, s^{\prime}\right)}$ with $s^{\prime}+m^{\prime}<s+m$ [2]. In the special case $m=1$, these relations give rise to the earlier proposed expansions of single-trace EYM amplitudes [3-5]. When the recursive expansions are applied repeatedly until there is no graviton and only one gluon trace, an arbitrary tree-level EYM amplitude is finally expressed as a combination of tree 
level color-ordered Yang-Mills (YM) ones. Such pure-YM expansions precisely coincide with the earlier studies on amplitudes with only a few gravitons and/or gluon traces [6-9].

The pure YM expansions of EYM amplitudes, together with the gauge invariance conditions of gravitons or the cyclic symmetries of gluon traces, induce nontrivial identities for color-ordered YM amplitudes [2]. These identities guaranteed the localities in the Britto-Cachazo-Feng-Witten (BCFW) [10, 11] proof of the recursive expansions $[2,3]$ and played a crucial role in the proof [12] of the equivalence between distinct approaches [13-15] to nonlinear sigma model amplitudes.

A prominent feature of the identities induced from multi-trace EYM amplitudes is that the coefficients therein generally contain factors of all the three types of Lorentz contractions $\epsilon \cdot \epsilon, \epsilon \cdot k$ and $k \cdot k$ where $\epsilon^{\mu}$ are half polarizations of gravitons and $k^{\mu}$ are external momenta. This is quite different from the known Kleiss-Kuijf (KK) [16] and Bern-CarrascoJohansson (BCJ) relations [17] whose coefficients at most involve $k \cdot k$ factors. Nevertheless, several clues imply that the identities induced from multi-trace EYM amplitudes can be related with BCJ relations: (i). First, the relationship between the identities induced from single-trace EYM amplitudes and BCJ relations have already been founded [1], while the multi-trace amplitudes can be obtained through replacing gravitons by gluon traces in an appropriate way (as pointed in [2]). (ii). Second, as demonstrated in [2], examples with a few gluon traces and gravitons provided evidence of the connection between the induced identities and BCJ relations. (iii). Third, the fact that color-kinematic duality [17], which is the underlaid structure of the BCJ relations for YM amplitudes, can be resulted from gauge invariance [18] also implies that the identities induced from gauge invariance can be related with BCJ relations.

In the current paper, we extend our discussions in [1] to multi-trace cases and show that all these identities induced from multi-trace EYM amplitudes can be expanded in terms of BCJ relations. The main idea is sketched as follows:

(i) Refined graphic rule. We first introduce a refined graphic rule ${ }^{1}$ for the coefficients in pure-YM expansions of multi-trace EYM amplitudes, where the three types of factors $\epsilon \cdot \epsilon, \epsilon \cdot k$ and $k \cdot k$ are presented by distinct types of lines (as already introduced in the study of single-trace case [1]) and a new type of line is invented to record the relative order of gluons in a gluon trace. The induced identities are then expressed through a summation over connected tree graphs which are built of the four types of lines, while color-ordered YM amplitudes corresponding to each graph can be collected in a proper way.

(ii) Skeletons and components. To relate the induced identities with BCJ relations, we split gluon traces in an appropriate way and then remove all the $k \cdot k$ lines from the graphs. After that, a physical graph (i.e. a graph defined by the refined graphic rule) turns to a disconnected one which is called skeleton and consists of disjoint components. The summation over all physical graphs is thus given by summing over

\footnotetext{
${ }^{1}$ Graphic approaches to scattering amplitudes can be found in many literatures including: graphic study on Cachazo-He-Yuan [19-22] formulas (see e.g. [23-30]), graphic construction for BCJ numerators [15] and the refined graphic rule for single-trace EYM amplitudes [1].
} 
all skeletons and summing over all the physical graphs corresponding to a given skeleton.

(iii) The final upper and lower blocks. For a given skeleton, the summation over all possible physical graphs can further be arranged by the following two steps: (a). Connect components via $k \cdot k$ lines properly such that the skeleton becomes a graph with only two disjoint maximally connected subgraphs, which are called the final upper and lower blocks, (b). Connect the final upper and lower blocks into a physical graph via a $k \cdot k$ line. Spurious graphs, which are not defined by the refined graphic rule, can also be introduced for a given configuration of the final upper and lower blocks. When associated with proper signs, all spurious graphs cancel out. Then the summation over all physical graphs for a given final upper and lower blocks can be reexpressed by a summation over all physical and spurious graphs.

(iv) Expressing an induced identity by BCJ relations. We finally find that all contributions of the physical and spurious graphs, corresponding to a given configuration of the final upper and lower blocks, together can be written as a combination of the graphbased BCJ relations [1] which have been proven to be combinations of the traditional BCJ relations [17]. ${ }^{2}$

The structure of this paper is the following. In section 2, we introduce a refined graphic rule for the expansion of multi-trace EYM amplitudes and then express the induced identities by this rule. We further show two examples in section 3 which support the fact that the contributions of all those graphs corresponding to a given skeleton can be written as a combination of graph-based BCJ relations. In section 4, the general pattern of skeletons and components are studied. We then provide the general construction of the final upper and lower blocks for a given skeleton. The pattern of spurious graphs is also discussed. We finally show how to express the contributions of all (physical and spurious) graphs for a given final upper and lower blocks in terms of the graph-based BCJ relations. This work is summarized in section 5. A review of the background knowledge, the proof of the splitting trace relation and the pattern of the signs for graphs are included in the appendix.

Convention of notations The notations in this paper are gathered as follows.

- Permutations and sets: permutations are denoted by boldface Greek letters: $\boldsymbol{\sigma}, \boldsymbol{\alpha}$, $\boldsymbol{\beta}, \boldsymbol{\gamma}, \boldsymbol{\zeta}$, etc. The $i$-th element in $\boldsymbol{\sigma}$ is denoted by $\sigma(i)$. The position of an element $a$ in $\boldsymbol{\sigma}$ is expressed by $\sigma^{-1}(a)$. The inverse permutation of elements in an ordered set $\boldsymbol{X}$ is denoted by $\boldsymbol{X}^{T}$. Shuffle permutations of two ordered sets $\boldsymbol{X}$ and $\boldsymbol{Y}$ are written as $\boldsymbol{X} \amalg \boldsymbol{Y}$. The number of elements in set $\boldsymbol{A}$ is presented by $|\boldsymbol{A}|$. We use $\boldsymbol{A} \backslash \boldsymbol{B}$ to denote the difference of the sets $\boldsymbol{A}$ and $\boldsymbol{B}$.

- Gravitons and gluon traces: gluon traces are denoted by boldface numbers 1, 2, $\ldots$, or boldface lowercase Latin letters $\boldsymbol{t}, \boldsymbol{i} \ldots$ If a trace $\boldsymbol{t}_{i}$ can be written as $\boldsymbol{t}_{i}=$

\footnotetext{
${ }^{2}$ In this paper, we distinguish the earlier proposed forms of BCJ relations (see [17, 31, 32]) from the graph-based BCJ relations [1] by the name traditional BCJ relations.
} 
$\left\{a_{i}, \boldsymbol{X}_{i}, b_{i}, \boldsymbol{Y}_{i}\right\}$ ( $\boldsymbol{X}_{i}$ and $\boldsymbol{Y}_{i}$ are the two ordered sets of gluons which are separated by the gluons $a_{i}$ and $\left.b_{i}\right)$, we define $\mathrm{KK}\left[\boldsymbol{t}_{i}, a_{i}, b_{i}\right] \equiv \boldsymbol{X}_{i} ш \boldsymbol{Y}_{i}^{T}$ and $(-1)^{\left|\boldsymbol{t}_{i}, a_{i}, b_{i}\right|} \equiv(-1)^{\left|\boldsymbol{Y}_{i}\right|}$. The notation $\mathbf{H}\left(\mathbf{H}=\left\{h_{1}, h_{2}, \ldots, h_{s}\right\}\right)$ stands for a graviton set with gravitons $h_{1}, \ldots, h_{s}$. The set of gravitons $h_{1}, \ldots, h_{s}$ and gluon traces $\mathbf{2}, \ldots, \boldsymbol{m}$ is denoted by $\mathcal{H}\left(\mathcal{H}=\left\{\mathbf{2}, \ldots, \boldsymbol{m}, h_{1}, \ldots, h_{s}\right\}\right)$. The multi-trace EYM amplitude with traces $\mathbf{1}=\{1, \ldots, r\}, \mathbf{2}, \ldots, \boldsymbol{m}$ and graviton set $\mathrm{H}$ is written as $A(1,2, \ldots, r|\mathbf{2}| \ldots \mid \boldsymbol{m} \| \mathrm{H})$.

- Graphs: graphs are denoted by $\mathcal{F}, \mathcal{G}$ or $\mathcal{T}$. The notation $\mathcal{G}^{\prime}$ stands for the skeleton of a graph $\mathcal{G}$. Reference order and root set are respectively expressed by $\mathrm{R}$ and $\mathcal{R}$. Components of a skeleton are given by $\mathscr{A}, \mathscr{B}, \mathscr{C} \ldots$, while a chain of components is denoted by $\mathbb{C H}$. The reference order of components is given by $\mathrm{R}_{\mathscr{C}}$. The final upper and lower blocks are respectively presented by $\mathscr{U}$ and $\mathscr{L}$ whose disjoint union is $\mathscr{U} \oplus \mathscr{L}$.

\section{Refined graphic rule for multi-trace EYM amplitudes and the induced identities}

In this section, we present a refined graphic rule, by which one expresses a tree level multi-trace EYM amplitude $A(1,2, \ldots, r|\mathbf{2}| \ldots \mid \boldsymbol{m} \| \mathrm{H})$ with $m$ gluon traces $\mathbf{1} \equiv$ $\{1,2, \ldots, r\}, \mathbf{2}, \ldots, \boldsymbol{m}$ and $s$ gravitons $\mathbf{H} \equiv\left\{h_{1}, h_{2}, \ldots, h_{s}\right\}$ in terms of $(m+s)$-point tree level color-ordered YM amplitudes:

$$
A(1,2, \ldots, r|\mathbf{2}| \ldots \mid \boldsymbol{m} \| \mathbf{H})=\sum_{\mathcal{F}} \mathcal{C}^{\mathcal{F}}\left[\sum_{\boldsymbol{\sigma}^{\mathcal{F}}} A\left(1, \boldsymbol{\sigma}^{\mathcal{F}}, r\right)\right],
$$

where we have summed over all possible connected tree graphs $\mathcal{F}$. Each graph $\mathcal{F}$ defines a coefficient $\mathcal{C}^{\mathcal{F}}$ and proper permutations $1, \boldsymbol{\sigma}^{\mathcal{F}}, r$ (of all elements in $\mathbf{1} \cup \mathbf{2} \cup \ldots \cup \boldsymbol{m} \cup \mathrm{H}$ ) according to the refined graphic rule. The expansion (2.1) is obtained by applying the recursive expansion (A.1) (see [2]) iteratively and it is essentially equivalent to the graphic expansion given in [2]. Two examples which are helpful for understanding the refined graphic rule are given in this section. We then provide two identities that are respectively induced by the gauge invariance condition of a graviton and the cyclic symmetry of a gluon trace.

\subsection{Refined graphic rule}

To illustrate the refined graphic rule for the expansion (2.1), it is helpful to consider the gluon trace $\mathbf{1}$ in the EYM amplitude $A(1,2, \ldots, r|\mathbf{2}| \ldots \mid \boldsymbol{m} \| \mathrm{H})$ as a special one and denote the set of other gluon traces $\mathbf{2}, \ldots, \boldsymbol{m}$ and all gravitons $h_{1}, \ldots, h_{s}$ by $\mathcal{H} \equiv\left\{\mathbf{2}, \ldots, \boldsymbol{m}, h_{1}, \ldots, h_{s}\right\}$. In the set $\mathcal{H}$, each gluon trace is always considered as a single object. The refined graphic rule is then expressed as follows.

Step-1. Define a reference order of elements in $\mathcal{H}$ as the following ordered set:

$$
\mathrm{R}=\left\{\mathcal{H}_{\rho(1)}, \mathcal{H}_{\rho(2)}, \ldots, \mathcal{H}_{\rho(l=m+s-1)}\right\}
$$




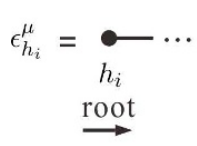

(a)

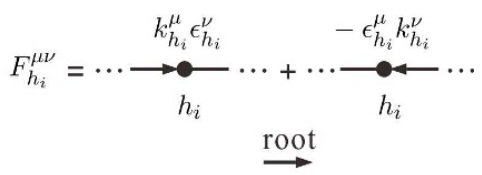

(b)

Figure 1. The graphs for a starting graviton and an internal graviton are correspondingly given by (b).

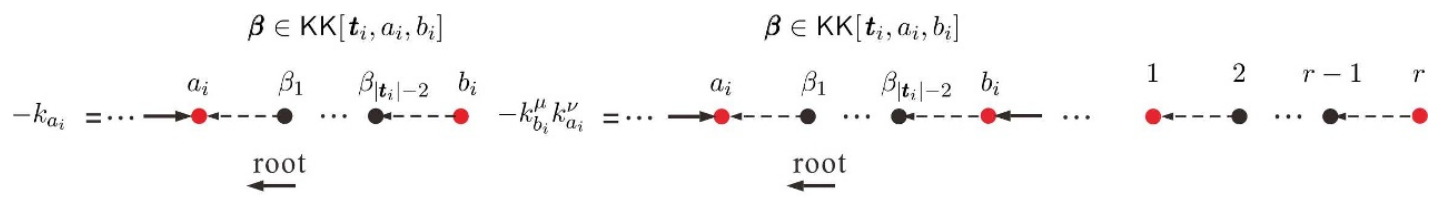

(a)

(b)

(c)

Figure 2. The starting trace and an internal trace of a chain are respectively presented by the graphs (a) and (b). The trace $\mathbf{1} \equiv\{1,2, \ldots, r\}$ is expressed by the structure (c). In this paper, the two end nodes of a trace are always colored red.

where each $\mathcal{H}_{i}$ stands for an element (i.e. a graviton or a gluon trace) of $\mathcal{H}$ and the position of $\mathcal{H}_{i}$ in $\mathrm{R}$ is called its weight. Apparently, $\mathcal{H}_{\rho(l=m+s-1)}$ is the highest-weight element in the reference order $(2.2)$, while $\mathcal{H}_{\rho(1)}$ is the lowest-weight one. We also define the root set $\mathcal{R}$ by collecting elements of the trace $\mathbf{1}$ :

$$
\mathcal{R} \equiv \mathbf{1} \backslash\{r\}=\{1,2, \ldots, r-1\},
$$

where the last element $r \in \mathbf{1}$ is always excluded. ${ }^{3}$

Step-2. Pick out the highest-weight element $\mathcal{H}_{\rho(l)}$ as well as other elements $j_{1}, \ldots, j_{u}$ (not necessary in the same relative order in $\mathrm{R}$ ) from the ordered set $\mathrm{R}$, then construct a chain towards an element $w$ in the root set $\mathcal{R}$ :

$$
\mathcal{H}_{\rho(l)} \rightarrow j_{u} \rightarrow \cdots \rightarrow j_{1} \rightarrow w .
$$

In the above chain, the $\mathcal{H}_{\rho(l)}, j_{1}, \ldots, j_{u}$ and $w$ are respectively mentioned as the starting element (graviton or trace), the internal elements (gravitons and/or traces) and the ending element (also mentioned as the root of the chain). The special case that a chain with no internal element $\mathcal{H}_{\rho(l)} \rightarrow w$ is allowed. The contribution of the chain (2.4) and the graphic expression are evaluated in the following way:

- Polarizations and momenta. Each half polarization $\epsilon^{\mu}$ of a graviton is expressed by a solid line connected to the graviton. The momentum $k^{\mu}$ of any node (graviton or gluon) is presented by a solid arrow line pointing to the node. Here, if an arrow points away from the direction of root, an extra minus should be dressed.

\footnotetext{
${ }^{3}$ If a set $B$ is a subset of $\boldsymbol{A}, \boldsymbol{A} \backslash \boldsymbol{B}$ is defined by moving all elements of $\boldsymbol{B}$ from $\boldsymbol{A}$.
} 


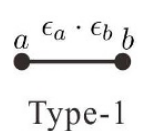

(a)

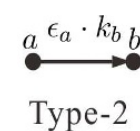

(b)

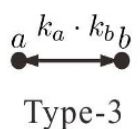

(c)

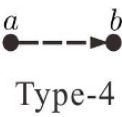

(d)

Figure 3. Four types of lines in the refined graphic rule for multi-trace EYM amplitudes

- Starting and internal gravitons. If the starting element $\mathcal{H}_{\rho(l)}$ is a graviton $h_{i}$, it contributes a half polarization $\epsilon_{h_{i}}^{\mu}$ presented by the structure figure 1 (a). If an internal element is a graviton $h_{i} \in\left\{j_{1}, \ldots, j_{u}\right\}$, it contributes a strength tensor $F_{h_{i}}^{\mu \nu} \equiv k_{h_{i}}^{\mu} \epsilon_{h_{i}}^{\nu}-k_{h_{i}}^{\nu} \epsilon_{h_{i}}^{\mu}$ and is expressed by the structure figure 1 (b).

- Starting and internal traces. To express a gluon trace $\boldsymbol{t}_{i}$, we select an ordered pair of gluons $\left\{a_{i}, b_{i}\right\}\left(a_{i}, b_{i} \in \boldsymbol{t}_{i}\right)$ where $a_{i}$ and $b_{i}$ play as the first and the last elements of the trace respectively. Then we arrange other gluons of $\boldsymbol{t}_{i}$ in a relative order $\boldsymbol{\beta} \in \mathrm{KK}\left[\boldsymbol{t}_{i}, a_{i}, b_{i}\right]$. Supposing that the trace can be written as $\boldsymbol{t}_{i}=\left\{a_{i}, \boldsymbol{X}, b_{i}, \boldsymbol{Y}\right\}$ where $\boldsymbol{X}$ and $\boldsymbol{Y}$ are the two ordered sets of gluons separated by $a_{i}$ and $b_{i}$, the $\mathrm{KK}\left[\boldsymbol{t}_{i}, a_{i}, b_{i}\right]$ is defined by permutations $\boldsymbol{X} \sqcup \boldsymbol{Y}^{T}$ ( $\boldsymbol{Y}^{T}$ is the inverse order of $\boldsymbol{Y}$ and $\boldsymbol{A} \sqcup \boldsymbol{B}$ for two ordered sets $\boldsymbol{A}, \boldsymbol{B}$ stands for the set of all permutations obtained by merging the two sets together with keeping the relative order of elements in each). Hence all gluons inside the trace are arranged in the relative order ${ }^{4}$

$$
a_{i}, \beta_{1}, \ldots, \beta_{\left|\boldsymbol{t}_{i}\right|-2}, b_{i}, \quad\left(\left\{\beta_{1}, \ldots, \beta_{\left|\boldsymbol{t}_{i}\right|-2}\right\} \equiv \boldsymbol{\beta} \in \mathrm{KK}\left[\boldsymbol{t}_{i}, a_{i}, b_{i}\right]\right) .
$$

Supposing $a_{i}$ is nearer to root than $b_{i}$, we draw a dashed arrow line between any two adjacent gluons in the permutation (2.5). Each arrow points towards the direction of the node $a_{i}$ as shown by figure 2 , thus it also points towards the root. If the trace $\boldsymbol{t}_{i}$ plays as the starting element $\mathcal{H}_{\rho(l)}$ of the chain (2.4), it is presented by the structure figure 2 (a) and contributes a $-k_{a_{i}}^{\mu}$ to the coefficient. If $\boldsymbol{t}_{i} \in\left\{j_{1}, \ldots, j_{u}\right\}$ is an internal trace, it should be expressed by the structure figure 2 (b) and contributes $\mathrm{a}-k_{b_{i}}^{\mu} k_{a_{i}}^{\nu}$. As shown by figure $2(\mathrm{c})$, the special trace $\mathbf{1}=\{1,2, \ldots, r\}$ is presented via connecting adjacent gluons by dashed arrow lines whose arrows point towards the direction of the first gluon 1 .

- Lorentz contractions and line styles. Contracting the Lorentz indices accompanying with adjacent elements, we get the contribution of the chain (2.4)

$$
\mathcal{E}_{\mathcal{H}_{\rho(l)}} \cdot \mathbb{F}_{j_{u}} \cdot \ldots \cdot \mathbb{F}_{j_{1}} \cdot k_{w}
$$

in which

$$
\begin{aligned}
\mathcal{E}_{x}^{\mu} & =\left\{\begin{array}{ccc}
\epsilon_{h_{i}}^{\mu} & \left(\text { if } x \text { is a graviton } h_{i}\right) \\
-k_{a_{i}}^{\mu} & \left(\text { if } x \text { is a gluon trace } \boldsymbol{t}_{i}\right)
\end{array}\right. \\
\mathbb{F}_{y}^{\mu \nu} & =\left\{\begin{array}{cl}
F_{h_{i}}^{\mu \nu} \equiv k_{h_{i}}^{\mu} \epsilon_{h_{i}}^{\nu}-k_{h_{i}}^{\nu} \epsilon_{h_{i}}^{\nu} & \text { (if } \left.y \text { is a graviton } h_{i}\right) \\
-k_{b_{i}}^{\mu} k_{a_{i}}^{\nu} & \text { (if } \left.y \text { is a gluon trace } \boldsymbol{t}_{i}\right)
\end{array}\right.
\end{aligned}
$$

\footnotetext{
${ }^{4}$ Here, the $l$-th element $\beta(l)$ in the permutation $\beta$ is labeled by $\beta_{l}$ for short.
} 
There are three types of lines figure 3 (a), (b) and (c), which are resulted by the Lorentz contraction (2.6) and correspond to $\epsilon \cdot \epsilon, \epsilon \cdot k$ and $k \cdot k$. Recalling that dashed arrow lines figure 3 (d) between gluons in a same trace have been introduced, we have four types of lines in all.

Redefine the ordered set $\mathrm{R}$ by removing the elements which have been used:

$$
\mathrm{R} \rightarrow \mathrm{R}^{\prime}=\mathrm{R} \backslash\left\{j_{1}, \ldots, j_{u}, \mathcal{H}_{\rho(l)}\right\} \equiv\left\{\mathcal{H}_{\rho\left(1^{\prime}\right)}, \mathcal{H}_{\rho\left(2^{\prime}\right)}, \ldots, \mathcal{H}_{\rho\left(l^{\prime}\right)}\right\}
$$

and redefine the root set $\mathcal{R}$ by:

$$
\mathcal{R} \rightarrow \mathcal{R}^{\prime}=\mathcal{R} \cup j_{1} \cup \cdots \cup j_{u} \cup \mathcal{H}_{\rho(l)} .
$$

Here each element in $\mathcal{R}$ is either a graviton or a gluon (this is different from the ordered set $\mathrm{R}$ where a trace is considered as a single element). If $j_{i}$ or $\mathcal{H}_{\rho(l)}$ in eq. (2.10) is a graviton, it always stands for a single-element set $\left\{j_{i}\right\}$ or $\left\{\mathcal{H}_{\rho(l)}\right\}$.

Step-3. Repeat the above step by using the new defined $\mathrm{R}$ and $\mathcal{R}$ until the ordered set $\mathrm{R}$ becomes empty. Then a fully connected tree graph $\mathcal{F}$ which is rooted at the gluon $1 \in \mathbf{1}$ is produced.

Step-4. For a given graph $\mathcal{F}$, the coefficient $\mathcal{C}^{\mathcal{F}}$ in eq. (2.1) can be read off as the product of all factors corresponding to the type- $1,-2$ and -3 lines (see figure 3 ). The sign associated with such a graph gets two distinct contributions (i). $(-1)^{\mathcal{N}(\mathcal{F})}$, where $\mathcal{N}(\mathcal{F})$ is the number of arrows pointing away from the gluon 1 ; (ii). Each trace $\boldsymbol{t}_{i}$ contributes a $(-1)^{\left|\boldsymbol{t}_{i}, a_{i}, b_{i}\right|}$ for given $a_{i}$ and $b_{i}$, where $\left|\boldsymbol{t}_{i}, a_{i}, b_{i}\right|$ is the number of elements in $\boldsymbol{Y}_{i}$ if the trace $\boldsymbol{t}_{i}$ can be written as $a_{i}, \boldsymbol{X}_{i}, b_{i}, \boldsymbol{Y}_{i}$.

Step-5. Collect amplitudes $A\left(1, \sigma^{\mathcal{F}}, r\right)$ for a given graph $\mathcal{F}$. In any graph $\mathcal{F}$, the gluons 1 (i.e. the root) and $r$ in the trace $\mathbf{1}=\{1,2, \ldots, r\}$ are always treated as the first and the last elements. Permutations $\boldsymbol{\sigma}^{\mathcal{F}}$ are determined as follows: (i). Two adjacent nodes $x$ and $y$ which are connected by a line (of any style) must live on a path towards the root 1 . If $x$ is nearer to 1 than $y$ on this path, we have $\left(\sigma^{\mathcal{F}}\right)^{-1}(x)<\left(\sigma^{\mathcal{F}}\right)^{-1}(y)$ where $\left(\sigma^{\mathcal{F}}\right)^{-1}(a)$ denotes the position ${ }^{5}$ of $a$. (ii). If there are several branches attached to a node, the relative order is defined by shuffling the branches together.

When summing over all possible graphs constructed by the above steps, (i.e., (i). summing over all graphs with given $\left\{a_{i}, b_{i}\right\}$ pairs and given permutations $\boldsymbol{\beta} \in \mathrm{KK}\left[\boldsymbol{t}_{i}, a_{i}, b_{i}\right]$ for all traces, (ii). summing over all possible permutations $\boldsymbol{\beta} \in \mathrm{KK}\left[\boldsymbol{t}_{i}, a_{i}, b_{i}\right]$ for given $\left\{a_{i}, b_{i}\right\}$ pairs in all traces, (iii). summing over all possible choices of the $\left\{a_{i}, b_{i}\right\}$ pairs for internal traces and all possible choices of $a_{i} \in \boldsymbol{t}_{i}, a_{i} \neq b_{i}$ for starting traces with fixed $b_{i}$ 's ${ }^{6}$ ), we

\footnotetext{
${ }^{5}$ This is understood as follows: assuming that the position of $a$ in $\boldsymbol{\sigma}^{\mathcal{F}}$ is $j$, we have $a=\sigma^{\mathcal{F}}(j)$, hence it is reasonable to define $j=\left(\sigma^{\mathcal{F}}\right)^{-1}(a)$.

${ }^{6}$ Notice that the refined graphic rule is given by applying the recursive expansion (A.1) iteratively. The $b_{i}$ of the starting trace in each step of recursive expansion can be chosen freely (see section A). This implies that the $b_{i}$ 's for the same trace, which plays as the starting element of different chains, can be chosen differently. In this paper, we fix the $b_{i} \in \boldsymbol{t}_{i}$ as the same one in all the graphs where the trace $\boldsymbol{t}_{i}$ plays as a starting element.
} 


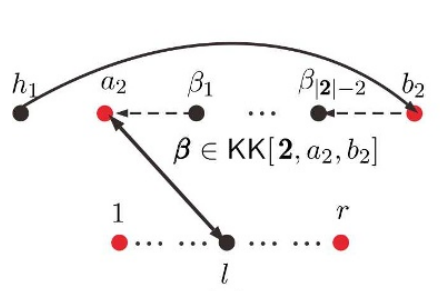

(a)

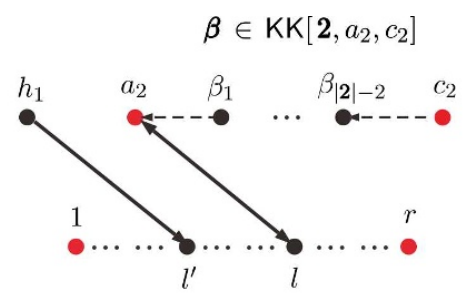

(b)

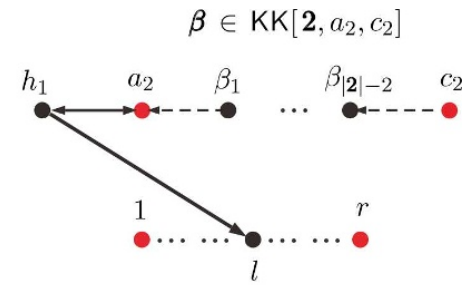

(c)

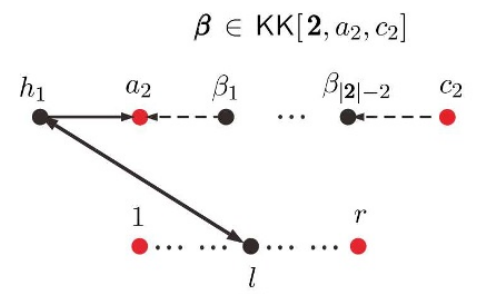

(d)

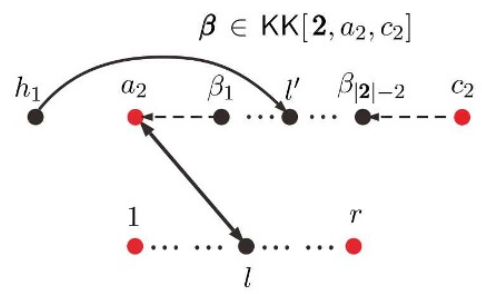

(e)

Figure 4. Typical graphs for the pure YM expansion of the amplitude $A\left(1,2 \ldots r \mid \mathbf{2} \| h_{1}\right)$. Graphs of structures (a), (b) and (c) contribute to the expansion with the reference order $\mathrm{R}=\left\{\mathbf{2}, h_{1}\right\}$. Graphs (b), (c), (d) and (e) contribute to the expansion with the reference order $\mathrm{R}=\left\{h_{1}, \mathbf{2}\right\}$.

finally arrive the expansion (2.1). In the coming subsection, we show a concrete example to explain this rule.

\subsection{Examples for the refined graphic rule}

Now we take the double-trace amplitude $A\left(1,2 \ldots r \mid \mathbf{2} \| h_{1}\right)$ with one graviton as an example. For this amplitude, the reference order can be chosen as either $\mathrm{R}=\left\{\mathbf{2}, h_{1}\right\}$ or $\mathrm{R}=\left\{h_{1}, \mathbf{2}\right\}$ in which the highest-weight element is respectively the graviton $h_{1}$ or the gluon trace $\mathbf{2}$. We study these two cases separately.

If $\mathrm{R}=\left\{\mathbf{2}, h_{1}\right\}$, the typical graphs are shown by figure 4 (a), (b) and (c). Correspondingly, these graphs contribute

$$
\begin{aligned}
& \mathcal{C}^{(\mathrm{a})}=-\left(\epsilon_{h_{1}} \cdot k_{b_{2}}\right)\left(k_{a_{2}} \cdot k_{l}\right), \\
& \boldsymbol{\sigma}^{(\mathrm{a})} \in\left\{2, \ldots, l,\{l+1, \ldots, r-1\} \amalg\left\{a_{2}, \beta_{1}, \ldots, \beta_{|\mathbf{2}|-2}, b_{2}, h_{1}\right\}\right\}, \\
& \mathcal{C}^{(\mathrm{b})}=-\left(\epsilon_{h_{1}} \cdot k_{l^{\prime}}\right)\left(k_{a_{2}} \cdot k_{l}\right), \\
& \boldsymbol{\sigma}^{(\mathrm{b})} \in\left\{2, \ldots, l^{\prime},\left\{h_{1}\right\} \amalg\left\{l^{\prime}+1, \ldots, l,\{l+1, \ldots, r-1\} \amalg\left\{a_{2}, \beta_{1}, \ldots, \beta_{|\mathbf{2}|-2}, c_{2}\right\}\right\}\right\}, \\
& \mathcal{C}^{(\mathrm{c})}=-\left(\epsilon_{h_{1}} \cdot k_{l}\right)\left(k_{a_{2}} \cdot k_{h_{1}}\right), \\
& \boldsymbol{\sigma}^{(\mathrm{c})} \in\left\{2, \ldots, l,\{l+1, \ldots, r-1\} \amalg\left\{h_{1}, a_{2}, \beta_{1}, \ldots, \beta_{|\mathbf{2}|-2}, c_{2}\right\}\right\},
\end{aligned}
$$

where the permutations $\boldsymbol{\beta}=\left\{\beta_{1}, \beta_{2}, \ldots, \beta_{|\mathbf{2}|-2}\right\}$ in $\boldsymbol{\sigma}^{(\mathrm{a})}$ and $\boldsymbol{\sigma}^{(\mathrm{b}),(\mathrm{c})}$ satisfy $\boldsymbol{\beta} \in \mathrm{KK}\left[\mathbf{2}, a_{2}, b_{2}\right]$ and $\beta \in \mathrm{KK}\left[\mathbf{2}, a_{2}, c_{2}\right]$ respectively. The signs caused by arrows pointing away from the root are absorbed into the coefficients $\mathcal{C}$ in eqs. (2.11), (2.12) and (2.13), but the signs $(-1)^{\left|\boldsymbol{t}_{2}, a_{2}, b_{2}\right|},(-1)^{\left|\boldsymbol{t}_{2}, a_{2}, c_{2}\right|}$ induced by the trace are not. Then the sum over all graphs 


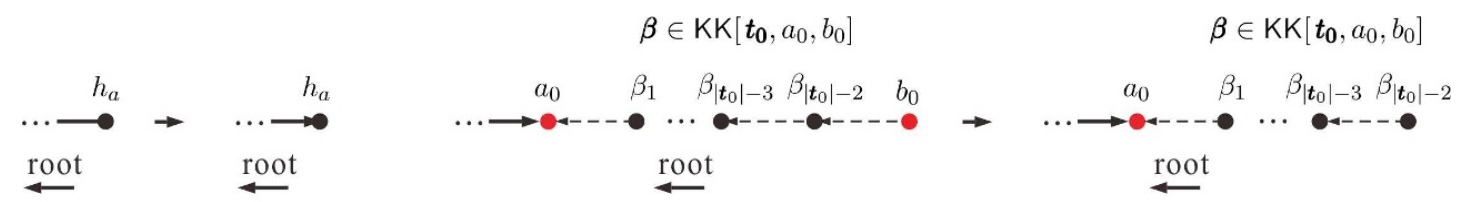

(a)

(b)

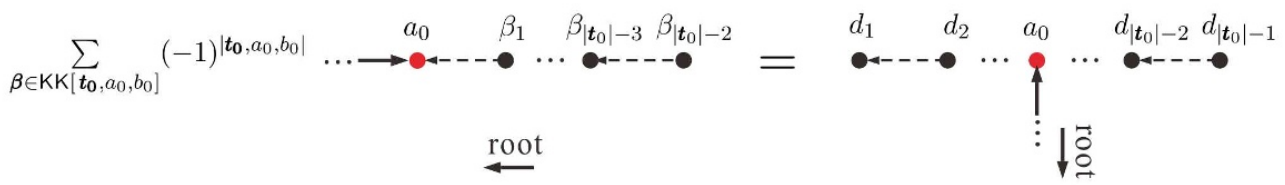

(c)

Figure 5. The induced identities (2.19) and (2.21) are obtained respectively by imposing the replacements (a) and (b) on the graphs in eq. (2.1) where the corresponding highest-weight elements are the graviton $h_{a}$ and the trace $\boldsymbol{t}_{0}$. The sum over all $\boldsymbol{\beta} \in \mathrm{KK}\left[\boldsymbol{t}_{0}, a_{0}, b_{0}\right]$ (with the sign $(-1)^{\left|\boldsymbol{t}_{0}, a_{0}, b_{0}\right|}$ ) on the r.h.s. of the replacement (b) can be further expressed by (c), where the full trace $\boldsymbol{t}_{0}$ is supposed to be $d_{1}, d_{2}, \ldots d_{j-1}, a_{0}=d_{j}, d_{j+1}, \ldots, d_{\left|\boldsymbol{t}_{0}\right|-1}, b_{0}=d_{\left|\boldsymbol{t}_{0}\right|}$.

in eq. (2.1) for the amplitude $A\left(1,2 \ldots r \mid 2 \| h_{1}\right)$ is given by

$$
\begin{gathered}
\sum_{l \in\{1, \ldots, r-1\}}\left[\widehat{\sum_{\left\{a_{2}, b_{2}\right\} \subset \mathbf{2}}} \sum_{\boldsymbol{\sigma}^{(\mathrm{a})}} \mathcal{C}^{(\mathrm{a})} A\left(1, \boldsymbol{\sigma}^{(\mathrm{a})}, r\right)\right. \\
+\overbrace{\substack{a_{2} \in \mathbf{2} \\
a_{2} \neq c_{2}\left(c_{2} \in \mathbf{2}\right)}}\left(\sum_{l^{\prime} \in\{1, \ldots, r-1\}} \sum_{\boldsymbol{\sigma}^{(\mathrm{b})}} \mathcal{C}^{(\mathrm{b})} A\left(1, \boldsymbol{\sigma}^{(\mathrm{b})}, r\right)+\sum_{\boldsymbol{\sigma}^{(\mathrm{c})}} \mathcal{C}^{(\mathrm{c})} A\left(1, \boldsymbol{\sigma}^{(\mathrm{c})}, r\right)\right)] .
\end{gathered}
$$

where the summation over $\boldsymbol{\beta} \in \mathrm{KK}\left[\mathbf{2}, a_{2}, b_{2}\right]\left(\boldsymbol{\beta} \in \mathrm{KK}\left[\mathbf{2}, a_{2}, c_{2}\right]\right)$ and the factor $(-1)^{\left|\boldsymbol{t}_{2}, a_{2}, b_{2}\right|}$ $\left((-1)^{\left|\boldsymbol{t}_{2}, a_{2}, c_{2}\right|}\right)$ corresponding to the trace $\mathbf{2}$ have been absorbed into the notation $\widetilde{\sum_{\left\{a_{2}, b_{2}\right\} \subset \mathbf{2}}}$

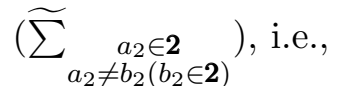

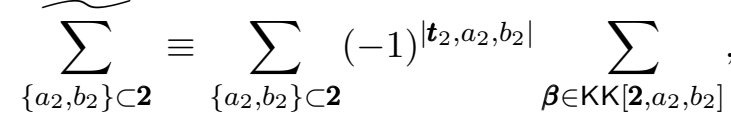

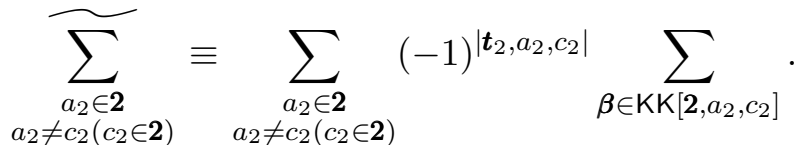

The $c_{2} \in \mathbf{2}$ in the second term of eq. (2.14) is arbitrarily fixed because the trace $\mathbf{2}$ plays as the starting trace in figure 4 (b) and (c).

If $\mathrm{R}=\left\{h_{1}, 2\right\}$, the typical graphs are given by figure 4 (b), (c), (d) and (e). The expression of figure 4 (b) and (c) are already shown by eq. (2.12) and eq. (2.13). The 
contributions from figure 4 (d) and (e) read

$$
\begin{aligned}
& \mathcal{C}^{(\mathrm{d})}=\left(k_{h_{1}} \cdot k_{l}\right)\left(\epsilon_{h_{1}} \cdot k_{a_{2}}\right), \\
& \boldsymbol{\sigma}^{(\mathrm{d})} \in\left\{2, \ldots, l,\{l+1, \ldots, r-1\} \amalg\left\{h_{1}, a_{2}, \beta_{1}, \ldots, \beta_{|\mathbf{2}|-2}, c_{2}\right\}\right\} \\
& \mathcal{C}^{(\mathrm{e})}=-\left(\epsilon_{h_{1}} \cdot k_{l^{\prime}}\right)\left(k_{a_{2}} \cdot k_{l}\right), \\
& \boldsymbol{\sigma}^{(\mathrm{e})} \in\left\{2, \ldots, l,\{l+1, \ldots, r-1\} \amalg\left\{a_{2}, \beta_{1}, \ldots, l^{\prime}=\beta_{j},\left\{\beta_{j+1}, \ldots, \beta_{|\mathbf{2}|-2}, c_{2}\right\} \amalg\left\{h_{1}\right\}\right\}\right\},
\end{aligned}
$$

where $l^{\prime}$ can be any element in the trace 2 . The sum over all graphs in eq. (2.1) for the amplitude $A\left(1,2 \ldots r \mid \mathbf{2} \| h_{1}\right)$ is then written as

$$
\begin{array}{r}
\sum_{l \in\{1, \ldots, r-1\}} \sum_{\substack{a_{2} \in \mathbf{2} \\
a_{2} \neq c_{2}\left(c_{2} \in 2\right)}}\left[\sum_{l^{\prime} \in\{1, \ldots, r-1\}} \sum_{\boldsymbol{\sigma}^{(\mathrm{b})}} \mathcal{C}^{(\mathrm{b})} A\left(1, \boldsymbol{\sigma}^{(\mathrm{b})}, r\right)+\sum_{\boldsymbol{\sigma}^{(\mathrm{c})}} \mathcal{C}^{(\mathrm{c})} A\left(1, \boldsymbol{\sigma}^{(\mathrm{c})}, r\right)\right. \\
\left.+\sum_{\boldsymbol{\sigma}^{(\mathrm{d})}} \mathcal{C}^{(\mathrm{d})} A\left(1, \boldsymbol{\sigma}^{(\mathrm{d})}, r\right)+\sum_{l^{\prime} \in \mathbf{2}} \sum_{\boldsymbol{\sigma}^{(\mathrm{e})}} \mathcal{C}^{(\mathrm{e})} A\left(1, \boldsymbol{\sigma}^{(\mathrm{e})}, r\right)\right]
\end{array}
$$

\subsection{Induced identities by refined graphs}

As pointed in [2], one can induce a nontrivial identity (see eq. (A.9) or eq. (A.10)) of EYM amplitudes by imposing each of the following conditions on the recursive expansion (A.1): (i). the gauge invariance condition of a graviton, (ii). the cyclic symmetry of a gluon trace. When the EYM amplitudes on the 1.h.s. of eq. (A.9) and eq. (A.10) are further expanded repeatedly according to eq. (A.1), we finally arrive identities for pure YM amplitudes. Such an identity can also be obtained by imposing the condition (i) or (ii) on eq. (2.1) straightforwardly. As shown in the appendix, we only need to study identities which are induced by conditions of the highest-weight element. Those identities induced by conditions of elements other than the highest-weight one are essentially treated as cases with a smaller $\mathcal{H}$ set.

If the highest-weight element $\mathcal{H}_{\rho(l)}$ in the reference order (2.2) is a graviton $h_{a}$, the gauge invariance condition of $h_{a}$ states that the expansion (2.1) vanishes when $\epsilon_{h_{a}}^{\mu}$ is replaced by $k_{h_{a}}^{\mu}$ :

$$
0=\left.\sum_{\mathcal{F}} \mathcal{C}^{\mathcal{F}}\right|_{\epsilon_{h_{a}} \rightarrow k_{h_{a}}}\left[\sum_{\boldsymbol{\sigma}^{\mathcal{F}}} A\left(1, \boldsymbol{\sigma}^{\mathcal{F}}, r\right)\right] .
$$

The corresponding graphs can be obtained by the replacement figure 5 (a). ${ }^{7}$

If the highest-weight element $\mathcal{H}_{\rho(l)}$ in the reference order $(2.2)$ is a gluon trace $\boldsymbol{t}_{0}$, the following identity is induced by the replacement

$$
\left\{a_{0}, \mathrm{KK}\left[\boldsymbol{t}_{0}, a_{0}, b_{0}\right], b_{0}\right\} \rightarrow\left\{a_{0}, \mathrm{KK}\left[\boldsymbol{t}_{0}, a_{0}, b_{0}\right]\right\},
$$

\footnotetext{
${ }^{7}$ Although, the arrow in figure 5 (a) points away from the root, we do not associate a minus sign with this graph, for an overall sign in the identity eq. (2.19) can always be neglected.
} 


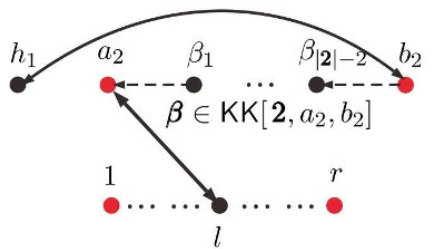

(a)

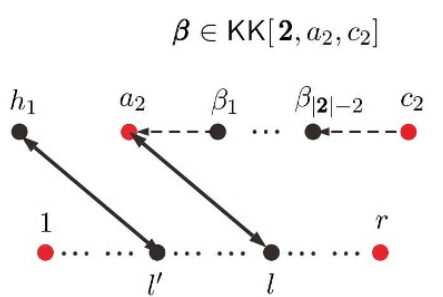

(b)

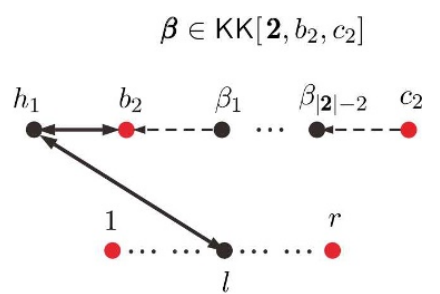

(c)

Figure 6. Typical graphs for the identity induced from the double-trace amplitude $A(1,2, \cdots$, $\left.r \mid \mathbf{2} \| h_{1}\right)$ with the reference order $\mathrm{R}=\left\{\mathbf{2}, h_{1}\right\}$. The trace $\mathbf{2}$ plays as an internal trace in (a) and a starting trace in (b), (c).

for any $a_{0} \in \boldsymbol{t}_{\mathbf{0}}\left(a_{0} \neq b_{0}\right)$ :

$$
0=\left.\sum_{\mathcal{F}} \mathcal{C}^{\mathcal{F}}\left[\sum_{\boldsymbol{\sigma}^{\mathcal{F}}} A\left(1, \boldsymbol{\sigma}^{\mathcal{F}}, r\right)\right]\right|_{\substack{\left\{a_{0}, \mathrm{KK}\left[t_{0}, a_{0}, b_{0}\right], b_{0}\right\} \\ \rightarrow\left\{a_{0}, \mathrm{KK}\left[\boldsymbol{t}_{0}, a_{0}, b_{0}\right]\right\}}}
$$

This identity is understood as follows: when we consider $\left\{a_{0}, \operatorname{KK}\left[\boldsymbol{t}_{0}, a_{0}, b_{0}\right]\right\}$ (for $a_{0} \in \boldsymbol{t}_{0}$ ) (which does not contain the fixed gluon $b_{0} \in \boldsymbol{t}_{0}$ ) as the highest-weight element $\mathcal{H}_{\rho(l)}$ (see figure 5 (b)) and then apply the refined graphic rule, the total contribution of the r.h.s. of eq. (2.1) must vanish. As stated in [2], this identity is essentially a result of the cyclic symmetry of the trace $\boldsymbol{t}_{0}$. It is worth pointing out an interesting property [2]: when we sum over $\boldsymbol{\beta} \in \mathrm{KK}\left[\boldsymbol{t}_{0}, a_{0}, b_{0}\right]$ with the sign $(-1)^{\left|\boldsymbol{t}_{0}, a_{0}, b_{0}\right|}$, the trace after the replacement figure 5 (b) becomes the r.h.s. of figure 5 (c) (where $\boldsymbol{t}_{0}$ is supposed to be $d_{1}, d_{2}, \ldots d_{j-1}, a_{0}=$ $\left.d_{j}, d_{j+1}, \ldots, d_{\left|\boldsymbol{t}_{0}\right|-1}, b_{0}=d_{|\boldsymbol{t}|_{0}}\right)$. In the coming sections, we always use the r.h.s. of figure 5 (c) to stand for the highest-weight element in the identity (2.21).

In the next section, we show that the identities (2.19) and (2.21) induced from the double-trace EYM amplitude $A\left(1, \cdots, r \mid \mathbf{2} \| h_{1}\right)$ can be expanded in terms of graph-based BCJ relations.

\section{Identities induced from $A\left(1, \cdots, r \mid 2 \| h_{1}\right)$ as combinations of BCJ re- lations}

We now take the identities induced from the amplitude $A\left(1, \cdots, r \mid \mathbf{2} \| h_{1}\right)$ as explicit examples and show that eq. (2.19) and eq. (2.21) can be written as combinations of graph-based BCJ relations (A.13) (thus traditional BCJ relations (A.12)). Critical features of these examples are further summarized.

\subsection{Example-1: the identity (2.19) induced from $A\left(1,2, \cdots, r \mid 2 \| h_{1}\right)$}

When the reference order is chosen as $\mathrm{R}=\left\{\mathbf{2}, h_{1}\right\}$, the highest-weight element is the graviton $h_{1}$. Thus the expansion (2.1) for the double-trace amplitude $A\left(1,2, \cdots, r \mid \mathbf{2} \| h_{1}\right)$ induces an identity (2.19) under the replacement $\epsilon_{h_{1}}^{\mu} \rightarrow k_{h_{1}}^{\mu}$. Typical graphs for this identity are given by figure 6 (a), (b) and (c) which are correspondingly obtained from figure 4 (a), 
(b) and (c) via the replacement figure 5 (a). The total contributions of these graphs are then written as

$$
\begin{aligned}
& T^{(\mathrm{a})}=\sum_{\boldsymbol{\sigma}^{(\mathrm{a})}}\left(k_{h_{1}} \cdot k_{b_{2}}\right)\left(-k_{a_{2}} \cdot k_{l}\right) A\left(1, \boldsymbol{\sigma}^{(\mathrm{a})}, r\right), \\
& T^{(\mathrm{b})}=\sum_{\boldsymbol{\sigma}^{(\mathrm{b})}}\left(k_{h_{1}} \cdot k_{l^{\prime}}\right)\left(-k_{a_{2}} \cdot k_{l}\right) A\left(1, \boldsymbol{\sigma}^{(\mathrm{b})}, r\right), \\
& T^{(\mathrm{c})}=\sum_{\boldsymbol{\sigma}^{(\mathrm{c})}}\left(k_{h_{1}} \cdot k_{l}\right)\left(-k_{b_{2}} \cdot k_{h_{1}}\right) A\left(1, \boldsymbol{\sigma}^{(\mathrm{c})}, r\right),
\end{aligned}
$$

where $\boldsymbol{\sigma}^{(\mathrm{a})}, \boldsymbol{\sigma}^{(\mathrm{b})}$ and $\boldsymbol{\sigma}^{(\mathrm{c})}$ are already presented in eq. (2.11), eq. (2.12) and eq. (2.13) respectively. Then the full expression of the r.h.s. of the identity (2.19) induced from $A\left(1, \cdots, r \mid \mathbf{2} \| h_{1}\right)$ is given by

$$
\left[\sum_{l \in\{1, \ldots, r-1\}} \widetilde{\sum_{\left\{a_{2}, b_{2}\right\} \subset 2}} T^{(\mathrm{a})}\right]+\left[\sum_{l, l^{\prime} \in\{1, \ldots, r-1\}} \widetilde{\sum_{\substack{a_{2} \in 2 \\ a_{2} \neq c_{2}\left(c_{2} \in \mathbf{2}\right)}}} T^{(\mathrm{b})}\right]+\left[\sum_{l \in\{1, \ldots, r-1\}} \sum_{\substack{b_{2} \in \mathbf{2} \\ b_{2} \neq c_{2}\left(c_{2} \in \mathbf{2}\right)}} T^{(\mathrm{c})}\right],
$$

where a summation notation with a tile is already defined by eq. (2.15).

To investigate the relationship between the induced identity and BCJ relations, we define the standard basis set for a gluon trace $\boldsymbol{i}$ by the set of permutations $\left\{\left\{b_{i}, \beta \in\right.\right.$ $\left.\left.\mathrm{KK}\left[\boldsymbol{i}, b_{i}, c_{i}\right], c_{i}\right\} \mid b_{i} \neq c_{i}, b_{i} \in \boldsymbol{i}\right\}$ for an arbitrarily fixed end node $c_{i} \in \boldsymbol{i}$ (i.e. we do not sum over the end node $c_{i}$ ). Graphically, any two adjacent nodes for a given permutation in the standard basis set $\left\{b_{i}, \boldsymbol{\beta} \in \mathrm{KK}\left[\boldsymbol{i}, b_{i}, c_{i}\right], c_{i}\right\}$ are connected by a dashed arrow line pointing towards the node $b_{i}$. A starting trace defined by the refined graphic rule is already expressed by the standard basis because an end node of this trace is already fixed. Moreover, all internal traces can be expanded by the standard basis, according to the following nontrivial property:

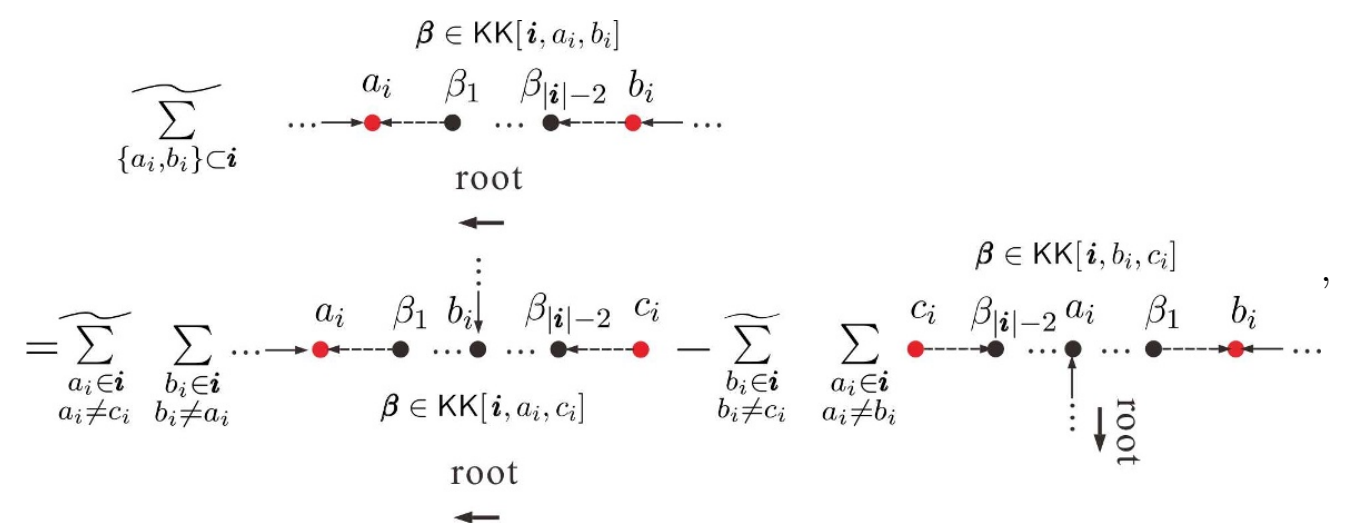

where each graph stands for its full contribution (including coefficients and amplitudes). The 1.h.s. of the above equation is an internal trace structure defined by the refined graphic rule. On the r.h.s., the trace $\boldsymbol{i}$ is expressed by the standard basis with one end $c_{i} \in \boldsymbol{i}$ fixed. The node $a_{i}\left(b_{i}\right)$ on both sides of eq. (3.5) must be connected to a same node outside the trace $\boldsymbol{i}$ via the same type of line (i.e., type- 2 or type- 3 line). The summation notations with 


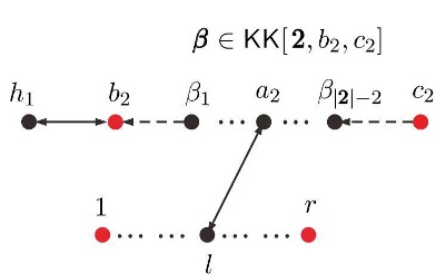

(a1)

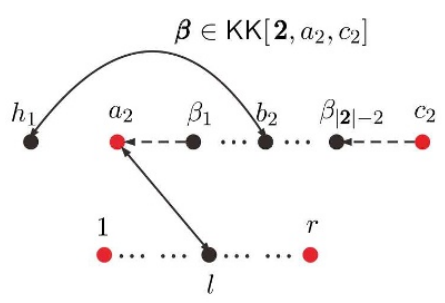

(a2)

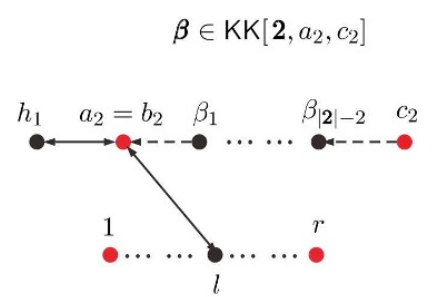

(a3)

Figure 7. When the identity (3.5) is applied, the contribution of figure 6 (a) splits into (a1) and (a2) which are expressed by standard basis. The graph (a1) comes from the second term of eq. (3.5), thus it must be associated with an extra minus. The graph (a3) is a spurious graph which cannot be directly obtained by the refined graphic rule and the identity (3.5). It can be considered as the $a_{i}=b_{i}$ supplement to both terms on the r.h.s. of eq. (3.5).

a tilde in eq. (3.5) was already defined by eq. (2.15). If a dashed arrow points away from the root, an extra minus should be dressed. We leave the proof of eq. (3.5) in appendix B.

Now we apply the relation (3.5) to the first term of eq. (3.4) for a given $l$. Since the $c_{i}$ in eq. (3.5) can be chosen arbitrarily, we just choose the $c_{i} \in \boldsymbol{i}$ (in this example $\boldsymbol{i}=\mathbf{2}$ ) as the fixed gluon $c_{2}$ in the trace 2 of figure 6 (b) and (c) for convenience. Then the summation $\widetilde{\sum}_{\left\{a_{2}, b_{2}\right\} \subset \mathbf{2}} T^{(\mathrm{a})}$ in the first term of eq. (3.4) splits into

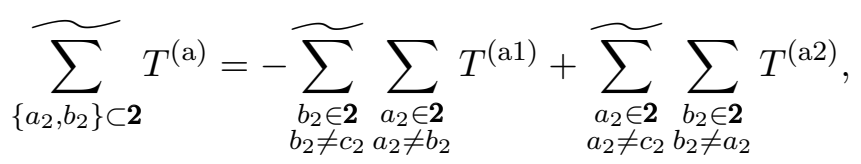

where $T^{(\mathrm{a} 1)}$ and $T^{(\mathrm{a} 2)}$ are corresponding to figure 7 (a1) and (a2). Substituting eq. (3.6) into eq. (3.4) and introducing the contribution of spurious graph figure 7 (a3) by $0=$ $T^{(\mathrm{a} 3)}-T^{(\mathrm{a} 3)}$, we rewrite eq. (3.4) as the sum of $I_{1}$ and $I_{2}$ which are respectively defined by

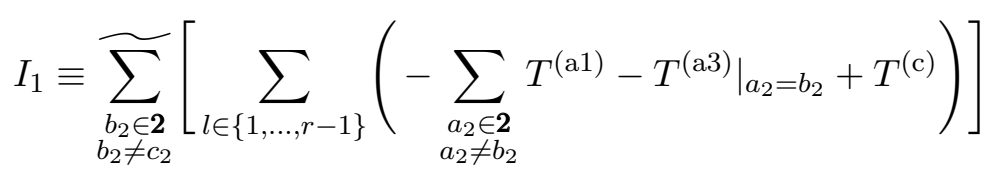

and

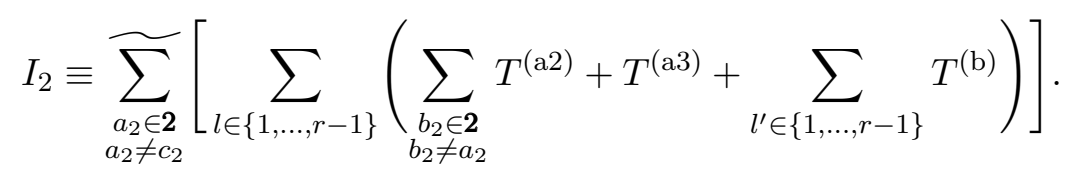

In the following, we analyze $I_{1}$ and $I_{2}$ in turn and prove that both of them can be expanded in terms of graph-based BCJ relations.

(i) It is easy to see the graphs figure 7 (a1), (a3) and figure 6 (c) corresponding to the three terms of eq. (3.7) can be reproduced by drawing a type-3 line between a gluon $l \in\{1,2, \ldots, r-1\}=\mathbf{1} \backslash\{r\}$ and a node $a \in \mathcal{T}_{1}$ where $\mathcal{T}_{1}$ is the tree structure figure 8. Particularly, $a$ is given by $a_{2} \in \mathbf{2}\left(a_{2} \neq b_{2}\right)$ for figure 7 (a1), $b_{2}$ for figure 7 (a3) and $h_{1}$ for figure 6 (c). The kinematic factors of figure 7 (a1), (a3) and figure 6 


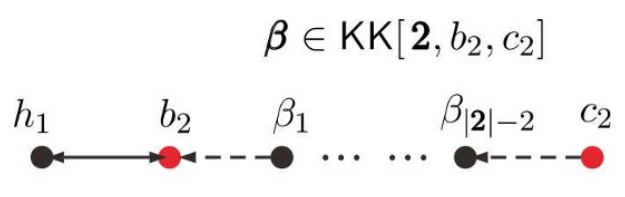

$\mathcal{T}_{1}$

Figure 8. The graphs figure 7 (a1), (a3) and figure 6 (c) can be reproduced by connecting the structure $\mathcal{T}_{1}$ with a gluon $l \in\{1,2, \ldots, r-1\}=\mathbf{1} \backslash\{r\}$ via a type-3 line. This further implies that the $I_{1}$ in eq. (3.7) can be expanded by graph-based BCJ relations (A.13) with $\mathcal{T}=\mathcal{T}_{1}$.

(c) can then be uniformly given by $\left(k_{h_{1}} \cdot k_{b_{2}}\right)\left(k_{a} \cdot k_{l}\right)$. Let us count the sign: given $b_{2} \in \mathbf{2}\left(b_{2} \neq c_{2}\right)$ in eq. (3.7), there is an overall sign $(-1)^{\left|2, b_{2}, c_{2}\right|}$ which has already been absorbed into the summation notation with a tilde $\widetilde{\sum}_{\substack{b_{2} \in \mathbf{2} \\ b_{2} \neq c_{2}}}$. The sign for each term inside the square brackets is collected as follows: (1). According to the refined graphic rule, any graph $\mathcal{F}$ with $\mathcal{N}(\mathcal{F})$ arrows (for both solid and dashed arrow lines) pointing away from the root 1 is associated with a sign $(-1)^{\mathcal{N}(\mathcal{F})} \cdot(2)$. Each of (a1) and (a3) has an extra sign $(-1)$. The above observations further lead to the following pattern:

- Once $a \in \mathcal{T}_{1}$ has been chosen, the sign is independent of the choice of $l \in$ $\{1,2, \ldots, r-1\}=\mathbf{1} \backslash\{r\}$ because neither $\mathcal{N}(\mathcal{F})$ nor the extra sign in eq. (3.7) relies on $l$. On another hand, two graphs with adjacent $a \in \mathcal{T}_{1}$ are associated with opposite signs.

- All permutations established by the graph with any given $a \in \mathcal{T}_{1}$ have the form

$$
\left\{1,\left.\sigma \in\{2, \ldots, r-1\} ш \mathcal{T}_{1}\right|_{a}, r\right\}
$$

Here $\left.\mathcal{T}_{1}\right|_{a}$ is introduced as the relative orders between nodes of the tree $\mathcal{T}_{1}$ when the node $a$ is considered as the leftmost one. According to the refined graphic rule, when we connect a type-3 line between $a \in \mathcal{T}_{1}$ and $l \in 1 \backslash\{r\}, l$ must be nearer to the root 1 than $a$. In other words, for a given permutation $\sigma$ in eq. (3.9), $l \in\{1, \ldots, r-1\}$ can be any node satisfying $\sigma^{-1}(l)<\sigma^{-1}(a)$. Then the total coefficient (which comes from the type-3 line between $a$ and $l$ ) for the permutation $\boldsymbol{\sigma}$ is collected as $-k_{a} \cdot Y_{a}(\boldsymbol{\sigma})$ where $Y_{a}^{\mu}(\boldsymbol{\sigma}) \equiv \sum_{\sigma^{-1}(l)<\sigma^{-1}(a)} k_{l}^{\mu}$ (the momentum of the root 1 is always included in this summation).

Altogether, $I_{1}$ in eq. (3.7) can be reexpressed by

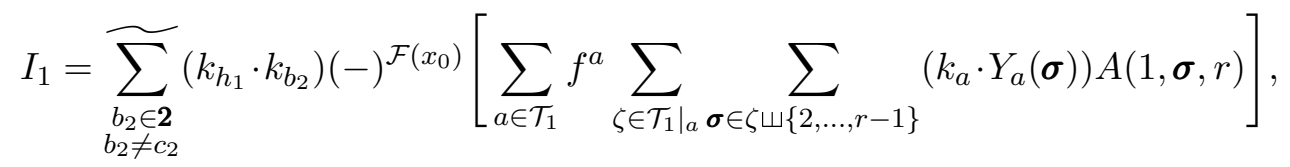

where $(-)^{\mathcal{F}\left(x_{0}\right)}$ denotes the sign for the graph $\mathcal{F}$ with $a=x_{0}\left(x_{0} \in \mathcal{T}_{1}\right)$. The $f^{a}$ for any $a \in \mathcal{T}_{1}$ is fixed as (i). $f^{x_{0}}=1$, (ii). $f^{x_{1}}=-f^{x_{2}}$ if $x_{1}$ and $x_{2}$ are two adjacent 


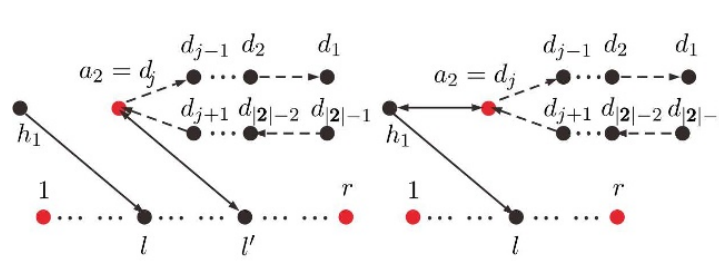

(a)

(b)

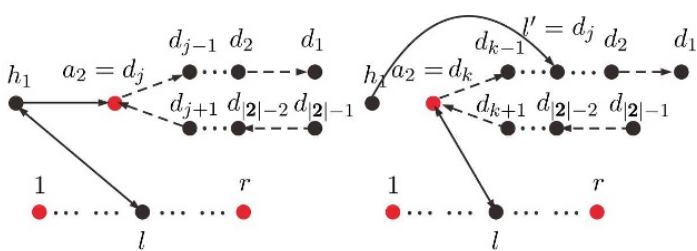

(c)

(d)

Figure 9. Typical graphs for the identity (2.21) induced from the double-trace amplitude $A\left(1,2, \cdots, r \mid \mathbf{2} \| h_{1}\right)$ with the reference order $\mathbf{R}=\left\{h_{1}, \mathbf{2}\right\}$. Here, gluons of the trace $\mathbf{2}$ are supposed to be in the cyclic order $d_{1}, d_{2}, \ldots, d_{|\mathbf{2}|}$ and the gluon $d_{|\mathbf{2}|}$ is the removed gluon $b_{0}$ in the induced identity (2.21).

nodes in $\mathcal{T}_{1}$. Therefore, the expression in the square brackets is just the 1.h.s. of the graph-based BCJ relation (A.13) which has been proven to be a combination of traditional BCJ relations (A.12) (see [1]).

(ii) For $I_{2}$, all the permutations established by the graphs figure 6 (b), figure 7 (a2) and (a3) have the form $\left\{1, \sigma \in\left\{h_{1}\right\} ш \gamma, r\right\}$, where

$$
\gamma \in\left\{2, \ldots, l,\{l+1, \ldots, r-1\} ш\left\{a_{2}, \mathrm{KK}\left[\mathbf{2}, a_{2}, c_{2}\right], c_{2}\right\}\right\} .
$$

Coefficient for each permutation $\boldsymbol{\sigma} \in\left\{h_{1}\right\} ш \boldsymbol{\gamma}$ is collected as $\left(-k_{a_{2}} \cdot k_{l}\right)\left(k_{h_{1}} \cdot Y_{h_{1}}(\boldsymbol{\sigma})\right)$. Hence $I_{2}$ turns to

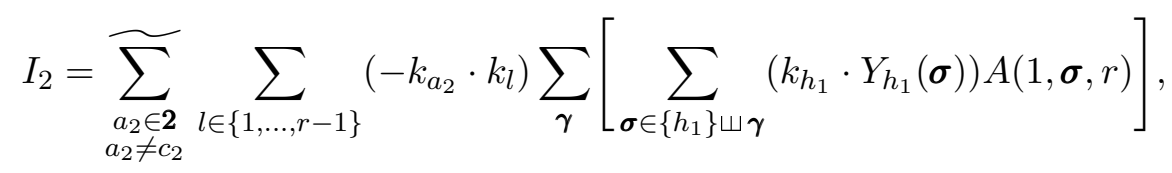

in which $\gamma$ satisfy eq. (3.11). Apparently, the expression in the square brackets is just the 1.h.s. of a special case of BCJ relation (A.12), which can also be understood as the graph-based BCJ relation (A.13) when the tree graph $\mathcal{T}$ is the single node $h_{1}$.

\subsection{Example-2: the identity (2.21) induced from $A\left(1,2, \cdots, r \mid 2 \| h_{1}\right)$}

When the reference order for the expansion (2.1) of the double-trace EYM amplitude $A\left(1,2, \cdots, r \mid \mathbf{2} \| h_{1}\right)$ is chosen as $\mathrm{R}=\left\{h_{1}, \mathbf{2}\right\}$ (i,e., the trace $\mathbf{2}$ is the highest-weight element), the cyclic symmetry of the trace $\mathbf{2}$ induces the identity (2.21) where typical graphs are presented as figure 4 (b)-(e). According to the discussions in section 2.3, we do the replacement figure 5 (b) and then figure 5 (c) on the graphs figure 4 (b)-(e). Thus graphs figure 9 (a)-(d) with the following contributions are obtained correspondingly:

$$
\begin{aligned}
T^{(\mathrm{a})} & =\sum_{\boldsymbol{\sigma}^{(\mathrm{a})}}\left(\epsilon_{h_{1}} \cdot k_{l}\right)\left(-k_{a_{2}} \cdot k_{l^{\prime}}\right) A\left(1, \boldsymbol{\sigma}^{(\mathrm{a})}, r\right), \\
T^{(\mathrm{b})} & =\sum_{\boldsymbol{\sigma}^{(\mathrm{b})}}\left(\epsilon_{h_{1}} \cdot k_{l}\right)\left(-k_{a_{2}} \cdot k_{h_{1}}\right) A\left(1, \boldsymbol{\sigma}^{(\mathrm{b})}, r\right), \\
T^{(\mathrm{c})} & =\sum_{\boldsymbol{\sigma}^{(\mathrm{c})}}\left(-\epsilon_{h_{1}} \cdot k_{a_{2}}\right)\left(-k_{h_{1}} \cdot k_{l}\right) A\left(1, \boldsymbol{\sigma}^{(\mathrm{c})}, r\right), \\
T^{(\mathrm{d})} & =\sum_{\boldsymbol{\sigma}^{(\mathrm{d})}}\left(\epsilon_{h_{1}} \cdot k_{l^{\prime}}\right)\left(-k_{a_{2}} \cdot k_{l}\right) A\left(1, \boldsymbol{\sigma}^{(\mathrm{d})}, r\right),
\end{aligned}
$$




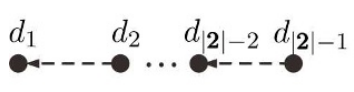

$\mathcal{T}_{3}$

(a)

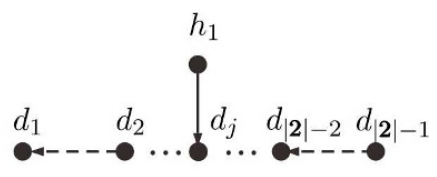

$\mathcal{T}_{4}$

(b)

Figure 10. The terms $I_{1}$ and $I_{2}$ in eq. (3.15) can be expanded in terms of graph-based BCJ relations (A.13), where the corresponding tree graphs $\mathcal{T}$ are chosen as the graph (a) and the graph (b).

in which,

$$
\begin{aligned}
& \boldsymbol{\sigma}^{(\mathrm{a})} \in\left\{2, \ldots, l,\left\{h_{1}\right\} \amalg\left\{l+1, \ldots, l^{\prime},\left\{l^{\prime}+1, \ldots, r-1\right\}\right.\right. \\
& \left.\left.\amalg\left\{a_{2}=d_{j},\left\{d_{j+1}, \ldots, d_{|\mathbf{2}|-1}\right\} \sqcup\left\{d_{j-1}, \ldots, d_{1}\right\}\right\}\right\}\right\}, \\
& \boldsymbol{\sigma}^{(\mathrm{b}),(\mathrm{c})} \in\left\{2, \ldots, l,\{l+1, \ldots, r-1\} \amalg\left\{h_{1}, a_{2}=d_{j},\left\{d_{j+1}, \ldots, d_{|2|-1}\right\} \amalg\left\{d_{j-1}, \ldots, d_{1}\right\}\right\},\right. \\
& \boldsymbol{\sigma}^{(\mathrm{d})} \in\left\{2, \ldots, l,\{l+1, \ldots, r-1\} \amalg\left\{a_{2}=d_{k},\left\{d_{k+1}, \ldots, d_{|\mathbf{2}|-1}\right\}\right.\right. \\
& \left.\left.\varpi\left\{d_{k-1}, \ldots, l^{\prime}=d_{j},\left\{h_{1}\right\} \amalg\left\{d_{j-1}, \ldots, d_{1}\right\}\right\}\right\}\right\} .
\end{aligned}
$$

Once all graphs are summed over, we arrive the r.h.s. of the induced identity eq. (2.21) for the amplitude $A\left(1,2, \cdots, r \mid \mathbf{2} \| h_{1}\right)$ :

$$
\underbrace{\sum_{l, l^{\prime} \in\{1, \ldots, r-1\}} \sum_{j=1}^{|2|-1} T^{(\mathrm{a})}+\sum_{l \in\{1, \ldots, r-1\}} \sum_{j=1}^{|2|-1}\left(T^{(\mathrm{b})}\right.}_{I_{1}}+\underbrace{\left.T^{(\mathrm{c})}\right)+\sum_{l \in\{1, \ldots, r-1\}} \sum_{j, k=1}^{|2|-1} T^{(\mathrm{d})}}_{I_{2}},
$$

where contributions of all graphs of the form figure 9 (a), (b) and (c), (d) were collected as $I_{1}$ and $I_{2}$ respectively. Now we prove that both $I_{1}$ and $I_{2}$ in eq. (3.15) can be expanded in terms of BCJ relations.

For the $I_{1}$ part in eq. (3.15), the summation over $j=1, \ldots,|2|-1$ is nothing but just the summation over all nodes $a_{2} \in \mathcal{T}_{3}$ where $\mathcal{T}_{3}$ is the tree graph figure 10 (a). All permutations established by the graphs figure 9 (a), (b) with a given $a_{2} \in \mathcal{T}_{3}$ and a given $l \in\{1, \ldots, r-1\}$ have the form

$$
\left\{1, \sigma \in\left(\left.\mathcal{T}_{3}\right|_{a_{2}}\right) \amalg \gamma, r\right\}
$$

Here, $\gamma \in\left\{2, \ldots, l,\left\{h_{1}\right\} ш\{l+1, \ldots, r-1\}\right\}$ and $\left.\mathcal{T}_{3}\right|_{a_{2}}$ denotes the relative orders of nodes in $\mathcal{T}_{3}$ when $a_{2}$ is considered as the leftmost one. Based on a similar discussion with the example-1 in section 3.1, we find the following patterns: (1). The coefficients $\left(k_{a_{2}} \cdot k_{l^{\prime}}\right)$ $\left(l^{\prime} \in\{1, \ldots, r-1\}\right.$ for figure 9 (a) and $l^{\prime}=h_{1}$ for figure 9 (b)) corresponding to a same $\boldsymbol{\sigma} \in\left(\left.\mathcal{T}_{3}\right|_{a_{2}}\right) \amalg \boldsymbol{\gamma}$ with different choices of $l^{\prime}$ are collected as $\left(-k_{a_{2}} \cdot Y_{a_{2}}(\boldsymbol{\sigma})\right) ;(2)$. Any two graphs, where $a_{2} \in \mathcal{T}_{3}$ are chosen as adjacent nodes, have opposite signs. Then the $I_{1}$ part 


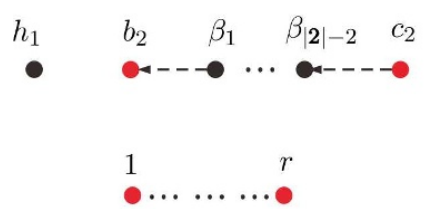

(a)

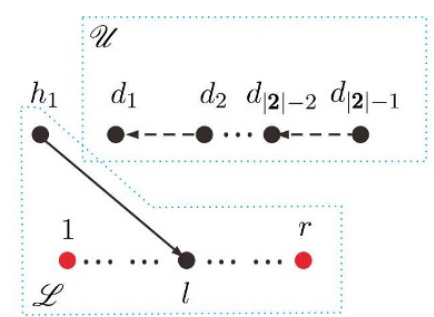

(b)

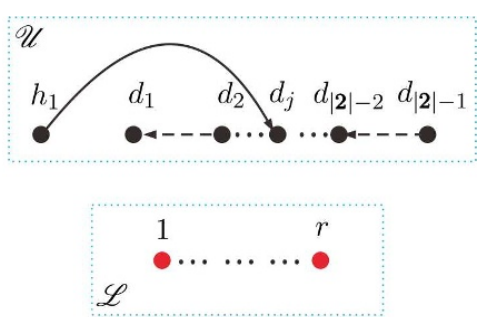

(c)

Figure 11. A typical skeleton in example-1 is presented as the graph (a) which consists of three components. Graphs (b) and (c) are possible structures of skeletons in example-2. The subgraphs $\mathscr{U}$ and $\mathscr{L}$ in each of (b) and (c) are correspondingly the final upper and lower blocks.

in eq. (3.15) is expressed by

$$
\begin{aligned}
& I_{1}=\sum_{l \in\{1, \ldots, r-1\}} \sum_{\gamma \in\left\{2, \ldots, l,\left\{h_{1}\right\}\right.}\left(\epsilon_{h_{1}} \cdot k_{l}\right)(-)^{\mathcal{F}\left(x_{0}\right)} \\
& \times\left[\sum_{a_{2} \in \mathcal{T}_{3}} f^{a_{2}} \sum_{\boldsymbol{\sigma} \in\left\{\left.\mathcal{T}_{3}\right|_{a_{2}} \omega \boldsymbol{\gamma}\right\}}\left(-k_{a_{2}} \cdot Y_{a_{2}}(\boldsymbol{\sigma})\right) A(1, \boldsymbol{\sigma}, r)\right] .
\end{aligned}
$$

Here $(-)^{\mathcal{F}\left(x_{0}\right)}$ is the sign for a graph with $a_{2}=x_{0} \in \mathcal{T}_{3}$. Since $(-)^{\mathcal{F}\left(x_{0}\right)}$ has been extracted as an overall sign, we have $f^{x_{0}}=1$. If $x_{1}, x_{2} \in \mathcal{T}_{3}$ are two adjacent nodes, we have $f^{x_{1}}=-f^{x_{2}}$. Obviously, the expression in the square brackets is nothing but (up to a total minus) the l.h.s. of a graph-based BCJ relation (A.13). As a result, $I_{1}$ is a combination of traditional BCJ relations.

The $I_{2}$ part in eq. (3.15) can be analyzed following a parallel discussion with $I_{1}$ but replacing the tree graph $\mathcal{T}_{3}$ by $\mathcal{T}_{4}$ (see figure $10(\mathrm{~b})$ ) for $d_{j} \in\left\{d_{1}, \ldots, d_{|\mathbf{2}|-1}\right\}$ and replacing $\gamma$ by $\{2, \ldots, r-1\}$ :

$$
I_{2}=\sum_{j=1}^{|2|-1}\left(\epsilon_{h_{1}} \cdot k_{d_{j}}\right)(-)^{\mathcal{F}\left(x_{0}\right)}\left[\sum_{a_{2} \in \mathcal{T}_{4}} f^{a_{2}} \sum_{\boldsymbol{\sigma} \in\left\{\left.\mathcal{T}_{4}\right|_{a_{2}} \uplus\{2, \ldots, r-1\}\right\}}\left(-k_{a_{2}} \cdot Y_{a_{2}}(\boldsymbol{\sigma})\right) A(1, \boldsymbol{\sigma}, r)\right],
$$

where $x_{0} \in \mathcal{T}_{4}$ and $f^{x_{0}}=1$. Again, the $f^{a_{2}}$ 's for adjacent choices of $a_{2}$ have the opposite signs. Up to a total sign, the expression in the square brackets is just the l.h.s. of graphbased BCJ relation (A.13), where the tree graph $\mathcal{T}_{4}$ for a given $j$ is figure $10(\mathrm{~b})$. Thus, we conclude that $I_{2}$ is a combination of BCJ relations.

\subsection{Common features of the examples}

Now let us extract some common features from the examples, which will be extended to general cases in the next section.

(i) Expressing traces by standard basis. In example-1, the trace $\mathbf{2}$ played as an internal trace in figure 6 (a) and a starting trace in either figure 6 (b) or (c). In the latter cases, the trace $\mathbf{2}$ was already expressed by the standard basis, i.e., one end of the trace, 


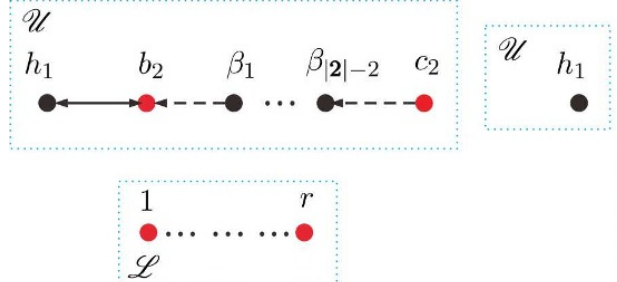

(a)

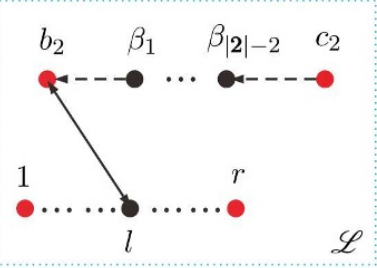

(b)

Figure 12. Typical configurations of the final upper and lower blocks $\mathscr{U}, \mathscr{L}$ for the skeleton figure 11 (a).

the gluon $c_{2}$, was fixed. In the former case, both ends of the trace $\mathbf{2}$ were not fixed (in other words both are summed over). In order to expand the trace $\mathbf{2}$ in figure 6 (a) by the standard basis, we have made used of the splitting trace relation (3.5), in which the fixed node was conveniently chosen as the same element (i.e. $c_{2}$ ) with that in figure $6(\mathrm{~b})$ and (c).

(ii) Skeletons and components. We define skeletons by removing all type-3 lines from the graphs where all traces, except the highest-weight element in $\mathrm{R}$ (if it is a trace), are already expressed by the standard basis. Since each graph defined by the refined graphic rule is a connected tree graph, its skeleton must be a disconnected graph. Each maximally connected subgraph of a skeleton is called a component. A typical skeleton in example-1 is given by figure 11 (a) (for $b_{2} \in \mathbf{2}, b_{2} \neq c_{2}$ ) which consists of three components. Skeletons in example-2 have two distinct structures, figure 11 (b) and (c), each of which has two components. From the examples, we can see any skeleton must have at least two components that involve the highest-weight element (graviton or trace) and the trace 1 respectively.

(iii) The final upper and lower blocks. Any graph in the examples can be reproduced by connecting a type-3 line between the final upper and lower blocks which are two mutually disjoint connected subgraphs and respectively contain the highest-weight element and the trace 1. In example-2, each of the skeletons figure 11 (b) and (c) already consists of only two disjoint connected subgraphs $\mathscr{U}$ and $\mathscr{L}$ which serve as the final upper and lower blocks. In example-1, there are three components in the skeleton figure 11 (a). A typical configuration of the final upper and lower blocks is constructed when we connect $b_{2}$ in figure 11 (a) to either (i). $h_{1}$ (see figure 12 (a)) or (ii). an element in $\{1, \ldots, r-1\}$ via a type-3 line (see figure $12(\mathrm{~b})$ ).

(iv) Physical and spurious graphs. For a given configuration of the final upper and lower blocks $\mathscr{U}$ and $\mathscr{L}$, we can connect two nodes $x \in \mathscr{U}$ and $y \in \mathscr{L} \backslash\{r\}$ (recalling that the gluon $r$ is always excluded) via a type-3 line. Then a fully connected graph is constructed. In example-2, the graphs figure 9 (a), (b) (and figure 9 (c), (d)) are reproduced by connecting the final upper and lower blocks in figure 11 (b) (and figure 11 (c)) via a type-3 line. Similarly, the graphs figure 7 (a1), figure 6 (c) (and figure 7 (a2), figure 6 (b)) in example-1 are constructed from figure 12 (a) (and (b)). 
All the graphs figure 6 (b), (c), figure 7 (a1), (a2) and figure 9 (a)-(d) are graphs in standard basis which are directly defined by the refined graphic rule. These graphs are called physical graphs. The spurious graph figure 7 (a3), which is not defined by the refined graphic rule, can be reproduced from either figure 12 (a) (connecting $b_{2} \in \mathscr{U}$ with $l \in \mathscr{L} \backslash\{r\}$ ) or figure 12 (b) (connecting $h_{1} \in \mathscr{U}$ with $b_{2} \in \mathscr{L} \backslash\{r\}$ ). In the former case, a minus sign is introduced so that the spurious graph constructed by distinct ways cancel with one another. Therefore, for any skeleton, the sum of all physical graphs can be given by (1). summing over all possible configurations of the final upper and lower blocks $\mathscr{U}, \mathscr{L},(\mathbf{2})$. for a given $\mathscr{U}$ and $\mathscr{L}$, connecting two nodes $x \in \mathscr{U}$ and $y \in \mathscr{L} \backslash\{r\}$ via a type-3 line and summing over all possible choices of $x, y$ (in other words summing over all possible physical and spurious graphs corresponding to $\mathscr{U}$ and $\mathscr{L}$ ).

(v) Induced identities as combinations of graph-based BCJ relations. A crucial observation is that the sum over all the graphs corresponding to a given configuration of the final upper and lower blocks $\mathscr{U}$ and $\mathscr{L}$ (i.e. either $I_{1}$ or $I_{2}$ in each example) is a combination of graph-based BCJ relations (A.13).

In the next section, we extend these observations to general cases and show that both identities (2.19) and (2.21) can be expanded in terms of BCJ relations.

\section{General study}

To investigate the general induced identities (2.19) and (2.21) in a unified way, we write them as

$$
0=\sum_{\mathcal{G}} \mathcal{C}^{\mathcal{G}} \sum_{\boldsymbol{\sigma}^{\mathcal{G}}} A\left(1, \boldsymbol{\sigma}^{\mathcal{G}}, r\right)
$$

where the graphs $\mathcal{G}$ are obtained by imposing the replacement figure 5 (a) or (b), which corresponds to eq. (2.19) or eq. (2.21), on the graphs $\mathcal{F}$ in eq. (2.1).

When we introduce skeletons $\mathcal{G}^{\prime}$ by deleting all type-3 lines from the graphs $\mathcal{G}$ and expressing all traces by standard basis according to eq. (3.5), eq. (4.1) is rearranged as

$$
0=\widetilde{\sum_{\mathcal{G}^{\prime}}} \mathcal{P}^{\left[\mathcal{G}^{\prime}\right]}\left[\sum_{\mathcal{G} \supset \mathcal{G}^{\prime}} \sum_{\sigma^{\mathcal{G}}}(-)^{\mathcal{G}} \mathcal{K}^{\left[\mathcal{G} \backslash \mathcal{G}^{\prime}\right]} A\left(1, \boldsymbol{\sigma}^{\mathcal{G}}, r\right)\right] .
$$

In the above equation, the summation notation $\widetilde{\sum}_{\mathcal{G}^{\prime}}$ means that all possible skeletons $\mathcal{G}^{\prime}$ are summed over and the signs $(-1)^{\left|\boldsymbol{t}_{i}, a_{i}, c_{i}\right|}$ and/or $(-1)^{\left|\boldsymbol{t}_{i}, b_{i}, c_{i}\right|}$ accompanying to the traces in each $\mathcal{G}^{\prime}$ are absorbed. The factor $\mathcal{P}^{\left[\mathcal{G}^{\prime}\right]}$ denotes the kinematic factor corresponding to the skeleton $\mathcal{G}^{\prime}$. Since a skeleton does not involve any type-3 line, $\mathcal{P}^{\left[\mathcal{G}^{\prime}\right]}$ only consists of $\epsilon \cdot \epsilon$ and $\epsilon \cdot k$ factors. In the expression inside the square brackets, all possible physical graphs $\mathcal{G}$ (i.e. graphs generated by refined graphic rule with all internal traces expressed by the standard basis) containing the skeleton $\mathcal{G}^{\prime}$ and all permutations $\sigma^{\mathcal{G}}$ for each graph $\mathcal{G}$ are summed over. The factor $\mathcal{K}^{\left[\mathcal{G} \backslash \mathcal{G}^{\prime}\right]}$ in eq. (4.2) stands for the product of all $k \cdot k$ factors that 


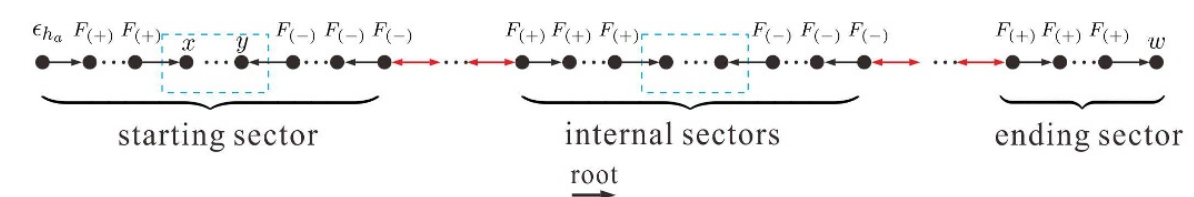

(a)

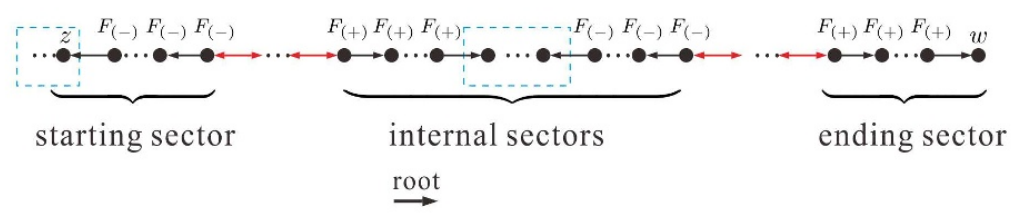

(b)

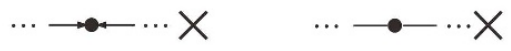

(c)

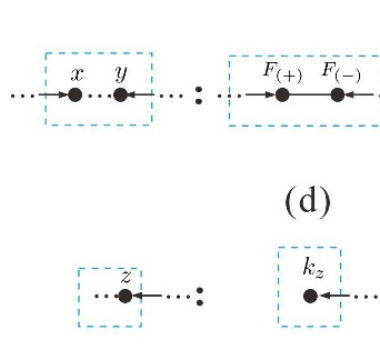

(g) (e)

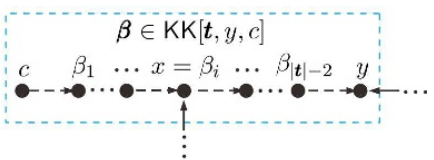

(f)

Figure 13. The graph (a) is a chain that does not involve the highest-weight element $\mathcal{H}_{\rho(l)}$ in the reference order R. The graph (b) is a chain involving the highest-weight element. Each node (except for the ending node $w$ ) outside the boxed structures in the chains (a) and (b) is a graviton and we define $F_{(+)}^{\mu \nu} \equiv k^{\mu} \epsilon^{\nu}, F_{(-)}^{\mu \nu} \equiv-\epsilon^{\nu} k^{\mu}$. The structures shown by the graph (c) are not allowed. Each boxed structure in the chain (a) and in an internal sector of the chain (b) can be (1). two gravitons connected together by a type-1 line (as shown by the graph (d)) or (2). a gluon trace in standard basis (as shown by (e) and (f) which correspond to the two terms in eq. (3.5)). The node $c$ in (e), (f) denotes the fixed node of a trace $t$. The boxed structure in the starting sector of (b) can be either a graviton (as shown by (g)) or a gluon trace $\boldsymbol{t} \rightarrow\left\{d_{1}, d_{2}, \ldots, d_{|\boldsymbol{t}|-1}\right\}$ (as shown by (h)).

are presented by the type- 3 lines in $\mathcal{G}$. Those signs caused by arrows pointing away from the root and the extra signs caused by the second term of eq. (3.5) are all collected as $(-)^{\mathcal{G}}$.

As observed in section 3.3, all physical graphs $\mathcal{G}$ involving a given skeleton $\mathcal{G}^{\prime}$ can be generated by connecting the components via type-3 lines in a proper way: (i). first generate all possible configurations of the final upper and lower blocks $\mathscr{U} \oplus \mathscr{L}$; (ii). then connect a type-3 line between two nodes $x \in \mathscr{U}$ and $y \in \mathscr{L} \backslash\{r\}$ appropriately. One should take care of the step (ii) because spurious graphs may also be produced. Nevertheless, the spurious graphs in fact all cancel out in the examples. Hence we suppose that the expression in the square brackets in eq. (4.2) can be generally written as

$$
I\left[\mathcal{G}^{\prime}\right] \equiv \sum_{\mathscr{U} \oplus \mathscr{L}} \mathcal{K}^{\left[\mathscr{U} \oplus \mathscr{L} \backslash \mathcal{G}^{\prime}\right]}\left[\sum_{\substack{x \in \mathscr{U} \\ y \in \mathscr{L} \backslash\{r\}}} \sum_{\boldsymbol{\sigma}^{\mathcal{G}}}(-)^{\mathcal{G}}\left(k_{x} \cdot k_{y}\right) A\left(1, \boldsymbol{\sigma}^{\mathcal{G}}, r\right)\right] .
$$




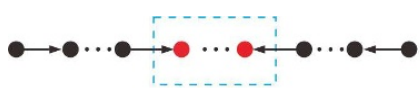

Type-I sector

(a)

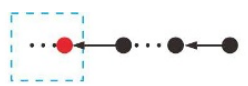

Type-II sector

(b)

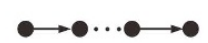

Type-III sector

(c)

Figure 14. There are three types of sectors (a), (b) and (c). The starting and internal sectors of the chain figure 13 (a) and the internal sectors of the chain figure 13 (b) have the same general structure (a). Such sectors are called type-I sectors. The starting sector of the chain figure 13 (b) is called the type-II sector and has the pattern (b). The ending sector of any chain has the structure (c) and is called a type-III sector.

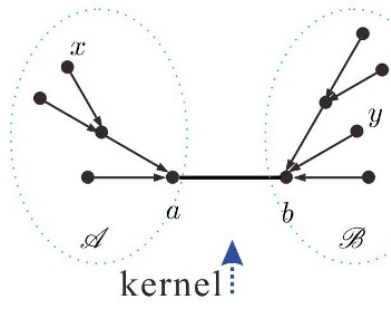

Type-IA component

(a)

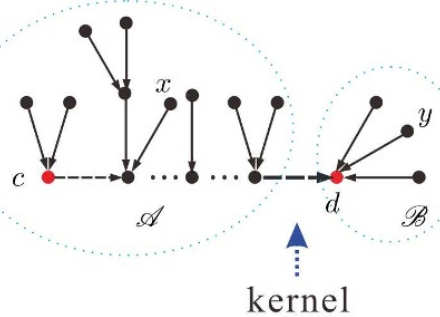

Type-IB component

(b)

Figure 15. Graph (a) is a type-IA component, while graph (b) is a type-IB component. The kernel of (a) is defined by the type- 1 line between nodes $a$ and $b$. The kernel of (b) is defined by the type- 4 line which is connected to the (unfixed) end node $d$. The nodes $x$ and $y$ in each graph are supposed to be the highest-weight nodes in the regions $\mathscr{A}$ and $\mathscr{B}$, respectively. If the weight of $x$ is higher than that of $y, \mathscr{A}$ and $\mathscr{B}$ are correspondingly the top and the bottom sides. Contrarily, if the weight of $y$ is higher than that of $x, \mathscr{A}$ becomes the bottom side while $\mathscr{B}$ the top.

Here, $\mathcal{K}^{\left[\mathscr{U} \oplus \mathscr{L} \backslash \mathcal{G}^{\prime}\right]}$ is the product of all $k \cdot k$ factors corresponding to the given configuration of the final upper and lower blocks $\mathscr{U}, \mathscr{L}$, while $k_{x} \cdot k_{y}$ is the factor corresponding to the type-3 line between $\mathscr{U}$ and $\mathscr{L}$. The first summation in eq. (4.3) is taken over all possible configurations of the final upper and lower blocks $\mathscr{U}, \mathscr{L}$ for the skeleton $\mathcal{G}^{\prime}$. In the square brackets, all choices of nodes $x \in \mathscr{U}$ and $y \in \mathscr{L} \backslash\{r\}$ as well as the permutations $\sigma^{\mathcal{G}}$ defined by the (physical or spurious) graph $\mathcal{G}$ (determined by $\mathscr{U}, \mathscr{L}, x$ and $y$ ) are summed over. The sign for the (physical or spurious) graph $\mathcal{G}$ is denoted by $(-)^{\mathcal{G}}$.

In this section, we study eq. (4.3) schematically. We first classify components of skeletons, then show how to construct the final upper and lower blocks from a given skeleton $\mathcal{G}^{\prime}$. After that, we show all spurious graphs cancel out. Thus the summation over all possible choices of $x \in \mathscr{U}$ and $y \in \mathscr{L} \backslash\{r\}$ is equivalent to summing over all possible physical graphs for given $\mathscr{U}$ and $\mathscr{L}$. At last, we demonstrate that the expression inside the square brackets in eq. (4.3) is a combination of BCJ relations.

\subsection{Skeletons and components}

When all type-3 lines (i.e. $k \cdot k$ factors) are removed, a graph $\mathcal{G}$ becomes a skeleton $\mathcal{G}^{\prime}$. To analyze possible structures of components which are maximally connected subgraphs of $\mathcal{G}^{\prime}$, we 


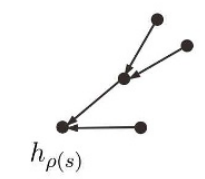

Type-IIA component

(a)

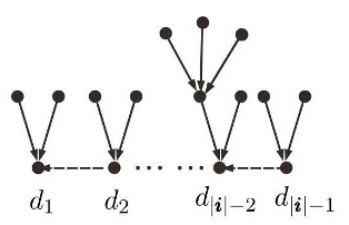

Type-IIB component

(b)

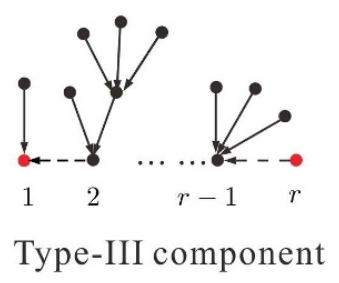

(c)

Figure 16. The component involving the highest-weight element $\mathcal{H}_{\rho(l)}$ is defined as (a). the typeIIA component if $\mathcal{H}_{\rho(l)}$ is a graviton $h_{\rho(s)}$, (b). the type-IIB component if $\mathcal{H}_{\rho(l)}$ is a gluon trace $\boldsymbol{t} \rightarrow\left\{d_{1}, \ldots, d_{|\boldsymbol{t}|-1}\right\}$. The component containing the trace $\mathbf{1}=\{1,2, \ldots, r-1, r\}$, as shown by the graph (c), is defined as the type-III component.

should look into the inner structure of a chain via expanding eq. (2.6) by eq. (2.7), eq. (2.8) and eq. (3.5). According to whether the starting node is the highest-weight element in the reference order $\mathrm{R}$ (defined in eq. (2.2)) or not, we carry out the discussion as follows:

(i) If the starting node of a chain is not the highest-weight element $\mathcal{H}_{\rho(l)}$, a graph corresponding to this chain can only have the general pattern figure 13 (a). Now we comment on crucial features of figure 13 (a): (1). Distinct sectors are separated by type-3 lines. The sector containing the starting (ending) node is called the starting (ending) sector. Other sectors between the starting and ending sectors are mentioned as internal sectors. (2). The possible structures inside the boxes in figure 13 (a) are given by figure 13 (d) (where two gravitons are connected by a type-1 line) and figure 13 (e), (f) (which involve a gluon trace expressed by standard basis). (3). If a chain figure 13 (a) contains only one sector, the sector must be the ending sector. (4). If the starting element of a chain is a gluon trace, there must be no graviton on the left hand side of the box in the starting sector, as shown by figure 13 (f). (5). Other structures of a chain are forbidden because the substructures in figure 13 (c) are not allowed by (eq. (2.6)) (in other words, $F_{h}^{\mu \nu} \equiv k_{h}^{\mu} \epsilon_{h}^{\nu}-\epsilon_{h}^{\mu} k_{h}^{\mu}$ for a graviton $h$ involves neither $k^{\mu} k^{\nu}$ nor $\left.\epsilon_{h}^{\mu} \epsilon_{h}^{\nu}\right)$.

(ii) If the starting node of a chain is the highest-weight element $\mathcal{H}_{\rho(l)}$, its corresponding graph must have the general pattern figure 13 (b), where the highest-weight element (graviton or trace) is already replaced according to figure 5 (a) (for a graviton) or figure 5 (b) and (c) (for a gluon trace). An important feature is the chain figure 13 (b) has at least two sectors, which follows from the fact that structures in figure 13 (c) are forbidden.

Having the above discussions, sectors of chains can be easily classified as figure 14 . In a full graph $\mathcal{G}$, nodes of any sector may play as the ending nodes of other chains. When all type-3 lines are removed, each sector in a skeleton thus can be attached by the type-3 sectors figure 14 (c) (or equivalently ending sectors) of other chains. Consequently, components in a skeleton can be classified by the following way. 
- Type-I component. A component consisting of a type-I sector figure 14 (a) and possible type-III sectors figure 14 (c) whose arrow lines point towards the type-I sector TypeI components can further be classified according to different structures inside the box of figure 14 (a): if the box contains the structure figure 13 (d), as shown by figure 15 (a), this component is called a type-IA component. Else, if the structure in the box is given by figure 13 (e) or (f), as shown by figure 15 (b), the component is called a type-IB component. We define the kernel of a type-I component by (i). the type- 1 line of a type-IA component (see figure 15 (a)), (ii). the type-4 line that is attached to the unfixed end node of the trace (in standard basis) inside a type-IB component (see figure 15 (b)). For a given reference order, any type-IA and -IB component is divided into two parts by the kernel: the part involving the highest-weight node (although a trace is considered as a single object in the reference order, the fixed node $c$ is always considered as the highest-weight node of this trace and it carries the weight of the full trace in the reference order) of this component is called the top side, while the opposite part is called the bottom side.

- Type-II component. A component consisting of a type-II sector figure 14 (a) and possible type-III sectors whose arrows point towards the type-II sector If the structure in the box of the type-II sector is figure 13 (g) (i.e. the highest-weight element is a graviton), this component is called a type-IIA component (see figure 16 (a)). If the structure in the box is a gluon trace figure $13(\mathrm{~h})$, the component is called a type-IIB component (see figure 16 (b)).

- Type-III component. A component consisting of the trace 1 and possible type-III sectors with arrows pointing towards the trace $\mathbf{1}$ (see figure 16 (c)).

Since the chain figure 13 (b) that is lead by the highest weight node has at least two sectors, a skeleton must at least contain two components: the type-II and the type-III components. In general, Type-I components may also be involved in a skeleton. All those graphs corresponding to a given skeleton are reproduced by connecting type-3 lines between components of a skeleton $\mathcal{G}^{\prime}$ in an appropriate way.

Multi-trace from single-trace. It is worth pointing out that the refined graphic rule given in section 2 can be obtained from the rule for identities induced from single-trace amplitudes (which was presented in [1]) by an appropriate replacement. Particularly, we consider a single-trace amplitude $A(1,2, \ldots, r \| \mathrm{H})$ where we have $s+m-1$ gravitons $\mathbf{H}=$ $\left\{h_{1}, \ldots, h_{s+m-1}\right\}$. When we replace $m-1$ gravitons, e.g., $h_{s+1}, h_{s+2}, \ldots, h_{s+m-1}$ by $m-1$ gluon traces $\mathbf{2}, \ldots, \boldsymbol{m}$, we get the multi-trace amplitude $A(1,2, \ldots, r|\mathbf{2}| \ldots \mid \boldsymbol{m} \| \mathrm{H})$ with $\mathbf{H}=$ $\left\{h_{1}, \ldots, h_{s}\right\}$. Such replacement is reflected in the refined graphic rule via replacing the reference order and chains with only gravitons by those with gravitons and/or gluon traces. The corresponding replacement for coefficients are given by

$$
\epsilon \cdot F \cdot F \cdot \ldots \cdot F \cdot k \stackrel{h_{s+1}, \ldots, h_{s+m-1} \rightarrow \mathbf{2}, \ldots, \boldsymbol{m}}{\longrightarrow} \mathcal{E} \cdot \mathbb{F} \cdot \mathbb{F} \cdot \ldots \cdot \mathbb{F} \cdot k,
$$

where $\epsilon, F$ are the half polarizations and strength tensors of gravitons, while $\mathcal{E}$ and $\mathbb{F}$ are generalized polarizations and generalized strength tensors for gravitons and/or gluon traces 
(see eq. (2.7) and eq. (2.8)). Graphically, this replacement is given by

figure 1 (a) $\rightarrow$ figure 2 (a), (if $h_{i}$ is replaced by $\boldsymbol{t}_{i}$ )

figure 1 (b) $\rightarrow$ figure 2 (b), (if $h_{i}$ is replaced by $\boldsymbol{t}_{i}$ ).

For identities induced from a single-trace amplitude, the highest-weight element (graviton) is further given by $k^{\mu}$ (see figure 5 (a)). In the corresponding multi-trace case, the highestweight element can either be a graviton (if it is not replaced by a trace) or be replaced by a gluon trace. The latter is described via replacing the node (the highest-weight graviton for identity induced from single-trace amplitude) by the r.h.s. of figure 5 (c).

Now we look into the inner structure of a chain by further expanding internal gravitons on the l.h.s. of eq. (4.4) according to $F^{\mu \nu}=k^{\mu} \epsilon^{\nu}-\epsilon^{\mu} k^{\nu} \equiv F_{(+)}^{\mu \nu}-F_{(-)}^{\mu \nu}$. On the r.h.s., internal gravitons are also expanded by $F_{(+)}^{\mu \nu}-F_{(-)}^{\mu \nu}$, while internal gluon traces are expanded according to the relation eq. (3.5). Although this expansion of gluon trace does not affect the tensor $\mathbb{F}^{\mu \nu}$ for a given $a_{i}$ and $b_{i}$ (see eq. (2.8)), it splits the graphs corresponding to the trace into the standard basis. Hence, the replacement $h_{i} \rightarrow \boldsymbol{i}$ (for an internal graviton $h_{i}$ ) is achieved graphically through replacing the first (second) graph in figure 1 (b) by the first (second) graph on the r.h.s. of eq. (3.5) (for a given $\left\{a_{i}, b_{i}\right\}$ ). It follows that the general chain structures figure 13 (a) and (b) are obtained from those chain structures for the single-trace case [1] (where only type-IA, type-IIA and type-III sectors are allowed) by incorporating more types of sectors: type-IB and type-IIB sectors which reflect structures of gluon traces. Consequently, the full classification of components in multi-trace cases can be given by enlarging the families of the type-I and the type-II components that were defined in [1]:

Type-I components $\rightarrow$ Type-IA or Type-IB components,

Type-II components $\rightarrow$ Type-IIA or Type-IIB components,

where the type-IIA and type-IIB components are respectively the highest-weight components of the identities (2.19) and (2.21). All the above discussions allow us to borrow some crucial conclusions from the single-trace case [1]:

(i) When keeping track of chains in the single-trace case [1], one can build all possible physical graphs corresponding to a skeleton by connecting type-3 lines between components properly (see appendix D in [1]). This construction can be immediately generalized to multi-trace cases by the enlargement (4.6).

(ii) As proved in [1] (see sections 6.1, 6.2 and appendix D of [1]), all the physical graphs in the single-trace case, which are corresponding to a given skeleton and are constructed by the above step, can be reproduced by (1). constructing the final upper and lower blocks, (2). connecting the final upper and lower blocks via a type-3 line appropriately. In multi-trace cases, we just follow the same construction rule but enlarging the type-I and type-II classes of components according to (4.6).

(iii) As pointed in section 6.3 of [1], in the single-trace case, the sum over all physical graphs which are produced by (ii) can be further written as the sum of all physical 
and spurious graphs (those graphs which are not directly constructed from the refined graphic rule). The latter all cancel out after summation. Again, the spurious graphs for single-trace induced identities can be straightforwardly extended to multi-trace cases by the help of (4.6) and they all cancel out (we have seen this cancellation by the examples in section 3 ).

In the coming two subsections, we display the construction rule of the final upper and lower blocks as well as the construction of physical and spurious graphs without a proof. In fact, all the proofs follow from discussions parallel with those in the single-trace case [1].

\subsection{The final upper and lower blocks}

Now we provide the general rule for constructing all possible configurations of the final upper and lower blocks corresponding to a given skeleton $\mathcal{G}^{\prime}$ :

- Step-1. For any skeleton $\mathcal{G}^{\prime}$, we define the reference order $\mathrm{R}_{\mathscr{C}}$ of all type-I components (including type-IA and type-IB components) by the relative order of the highest-weight nodes therein. In other words, the weight (i.e. the position in $\mathrm{R}_{\mathscr{C}}$ ) of a component inherits from its highest-weight node. For example, suppose there are three type-I components (IA and/or IB) $\mathscr{C}_{1}, \mathscr{C}_{2}$, and $\mathscr{C}_{3}$ with the corresponding highest-weight nodes (graviton or a gluon) $a_{1}, a_{2}$ and $a_{3}$. If the weights $W_{a_{i}}$ have the relation $W_{a_{2}}<W_{a_{1}}<W_{a_{3}}$, the reference order of these components is then given by the ordered set $\mathrm{R}_{\mathscr{C}}=\left\{\mathscr{C}_{2}, \mathscr{C}_{1}, \mathscr{C}_{3}\right\}$. We further define the upper block $\mathscr{U}$ and lower block $\mathscr{L}$ as the components respectively containing the highest-weight element (graviton or trace) and the trace 1. At the beginning, the upper and the lower blocks are nothing but the type-II and the type-III components.

- Step-2. Supposing the reference order of components is $\mathrm{R}_{\mathscr{C}}=\left\{\mathscr{C}_{1}, \mathscr{C}_{2}, \ldots, \mathscr{C}_{N}\right\}$, pick out the highest-weight component $\mathscr{C}_{N}$ as well as arbitrary components $\mathscr{C}_{a_{1}}, \mathscr{C}_{a_{2}}, \ldots$, $\mathscr{C}_{a_{i}}$ (the relative order of these components is not necessary the same relative order in $\mathrm{R}_{\mathscr{C}}$ ). Construct a chain of components towards either the upper block or the lower block as follows

$$
\begin{aligned}
\mathbb{C H}=\left[\left(\mathscr{C}_{N}\right)_{t},\left(\mathscr{C}_{N}\right)_{b}\right. & \leftrightarrow\left(\mathscr{C}_{a_{i}}\right)_{t(\text { or } b)},\left(\mathscr{C}_{a_{i}}\right)_{b(\text { or } t)} \\
\leftrightarrow & \left.\cdots \leftrightarrow\left(\mathscr{C}_{a_{1}}\right)_{t(\text { or } b)},\left(\mathscr{C}_{a_{1}}\right)_{b(\text { or } t)} \leftrightarrow \mathscr{U} \text { or } \mathscr{L} \backslash\{r\}\right] .
\end{aligned}
$$

Here the subscripts $t$ and $b$ respectively denote the top and bottom sides of a type-I component, which are separated by a comma. ${ }^{8}$ The double arrow line ' $\leftrightarrow$ ' between two components stands for the type-3 line (i.e. $k \cdot k$ ), which connects any two nodes belonging to the corresponding regions. For example, if the chain of components has the form $\left[\left(\mathscr{C}_{N}\right)_{t},\left(\mathscr{C}_{N}\right)_{b} \leftrightarrow\left(\mathscr{C}_{a_{i}}\right)_{t},\left(\mathscr{C}_{a_{i}}\right)_{b} \leftrightarrow \cdots\right]$, the two ends $x$ and $y$ of the type-3 line between the components $\mathscr{C}_{N}$ and $\mathscr{C}_{a_{i}}$ must belong to $\left(\mathscr{C}_{N}\right)_{b}$ and $\left(\mathscr{C}_{a_{i}}\right)_{t}$ respectively. After this step, we redefine the reference order of components as well as the upper

\footnotetext{
${ }^{8}$ Notations here are slightly different from those in [1].
} 


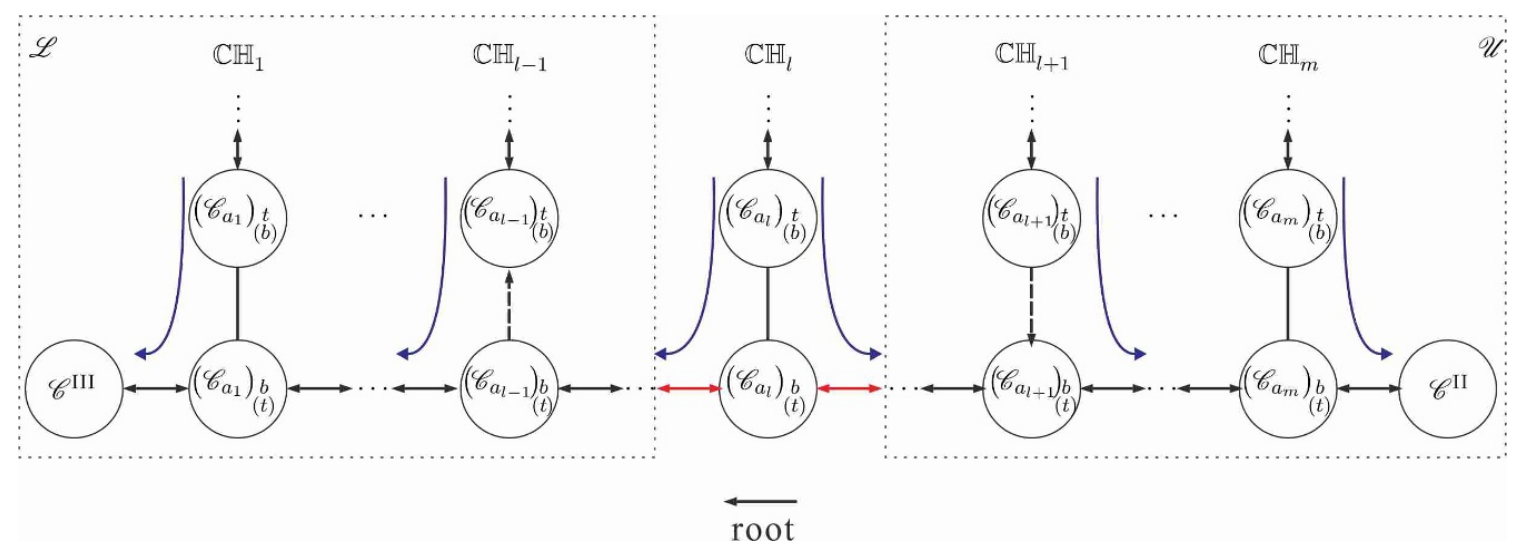

Figure 17. A typical spurious graph where the path starting from the highest-weight element and ending at the root 1 passes through some single sides of type-I (IA or IB) components $\mathscr{C}_{a_{1}}, \mathscr{C}_{a_{2}}, \ldots, \mathscr{C}_{a_{m}}$ (which are called spurious components).

and lower blocks by:

$$
\begin{aligned}
\mathrm{R}_{\mathscr{C}} & \rightarrow \mathrm{R}_{\mathscr{C}}^{\prime}=\mathrm{R} \mathscr{C} \backslash\left\{\mathscr{C}_{N}, \mathscr{C}_{a_{i}}, \ldots, \mathscr{C}_{a_{1}}\right\}=\left\{\mathscr{C}_{1}^{\prime}, \mathscr{C}_{2}^{\prime}, \ldots, \mathscr{C}_{N}^{\prime}\right\}, \\
\mathscr{U} & \rightarrow \mathscr{U}^{\prime}=\mathscr{U} \cup\left\{\mathscr{C}_{N}, \mathscr{C}_{a_{i}}, \ldots, \mathscr{C}_{a_{1}}\right\}, \\
\mathscr{L} & \rightarrow \mathscr{L}^{\prime}=\mathscr{L} \quad(\text { if } \mathbb{C H} \text { was attached to } \mathscr{U}) \\
\mathscr{L} & \rightarrow \mathscr{L}^{\prime}=\mathscr{L} \cup\left\{\mathscr{C}_{N}, \mathscr{C}_{a_{i}}, \ldots, \mathscr{C}_{a_{1}}\right\}, \\
\mathscr{U} & \rightarrow \mathscr{U}^{\prime}=\mathscr{U} \quad(\text { if } \mathbb{C H} \text { was attached to } \mathscr{L}) .
\end{aligned}
$$

- Step-3. Repeating step-2 with the new defined $\mathrm{R}_{\mathscr{C}}, \mathscr{U}$ and $\mathscr{L}$ iteratively until the ordered set $\mathrm{R}$ becomes empty, we get a graph with only two mutually disjoint subgraphs: the final upper and lower blocks $\mathscr{U}$ and $\mathscr{L}$.

All possible configurations of the final upper and lower blocks are produced by the above steps. The final upper and lower blocks for the examples in section 3 are precisely reproduced by this rule (see figure 12 (a), (b) for example-1 and figure 11 (b), (c) for example-2).

\subsection{Physical and spurious graphs}

For a given configuration of the final upper and lower blocks $\mathscr{U}$ and $\mathscr{L}$ which are constructed previously, a fully connected graph $\mathcal{G}$ in eq. (4.3) is produced by connecting arbitrary two nodes $x \in \mathscr{U}$ and $y \in \mathscr{L} \backslash\{r\}$ via a type-3 line. As pointed in section 3, such a graph can be either a physical graph or a spurious one. We have already stated that physical and spurious graphs can be obtained from those in single-trace case [1] by the enlargement (4.6). As a result, a spurious graph has the structure figure 17, where the chain starting form the highest-weight element (graviton for the identity (2.19) and gluon for the identity (2.21)) and ending at the root 1 passes through single sides of some type-IA and/or type-IB components $\mathscr{C}_{a_{1}}, \mathscr{C}_{a_{2}}, \ldots, \mathscr{C}_{a_{m}}$ which respectively belong to the chains of components $\mathbb{C H}_{1}, \ldots, \mathbb{C H}_{m}$. 
In order to display more details of spurious graphs, we define the weight $W_{i}$ of a chain $\mathbb{C H}_{a_{i}}$ by the weight of the starting component (equivalently the highest-weight component) of $\mathbb{C H}_{a_{i}}$. As pointed in [1], if the lowest-weight chain among $\mathbb{C H}_{1}, \ldots, \mathbb{C H}_{m}$ in figure 17 is $\mathbb{C H}_{l}$, we must have $W_{1}>\cdots>W_{l-1}>W_{l}$ and $W_{l}<W_{l+1}<\cdots<W_{m}$. Following a discussion which is parallel with that in [1], we conclude that all the chains $\mathbb{C H}_{1}, \ldots, \mathbb{C H}_{l-1}$ (and structures attached to them) belong to the final lower block $\mathscr{L}$, while $\mathbb{C H}_{l+1}, \ldots, \mathbb{C H}_{m}$ (and structures attached to them) belong to the final upper block $\mathscr{U}$. Only the lowestweight chain $\mathbb{C H}_{l}$ (among $\mathbb{C H}_{l+1}, \ldots, \mathbb{C H}_{m}$ ) can live in either $\mathscr{L}$ or $\mathscr{U}$. Correspondingly, the type-3 line (colored by red in figure 17) on either the l.h.s. or the r.h.s. of $\mathscr{C}_{a_{l}}$ is considered as the one between the final upper and lower blocks. Thus a given spurious graph is corresponding to two distinct configurations of $\mathscr{U}$ and $\mathscr{L}$. In other words, all spurious graphs must appear in pairs! This fact allows us to associate a pair of spurious graphs with opposite signs so that all spurious graphs cancel out.

By the help of the above discussion, we now determine the sign $(-)^{\mathcal{G}}$ in eq. (4.3) for a (physical or spurious) graph $\mathcal{G}$ :

(i) As proposed in [1], a graph is accompanied by a $\operatorname{sign}(-1)^{S\left(\mathscr{U}_{x}\right)}$, where $S\left(\mathscr{U}_{x}\right)$ is the number of spurious components living in the final upper block $\mathscr{U}$ for a given $x \in \mathscr{U}$ in eq. (4.3). For the typical spurious graph figure 17, $S\left(\mathscr{U}_{x}\right)=(-1)^{m-l+1}$, if $\mathscr{C}_{a_{l}}$ belongs to the final upper block $\mathscr{U}$, while $S\left(\mathscr{U}_{x}\right)=(-1)^{m-l}$, if $\mathscr{C}_{a_{l}}$ belongs to the final lower block $\mathscr{L}$. Hence spurious graphs cancel in pairs precisely.

(ii) Another sign which should be taken into account is introduced by the relation eq. (3.5). Particularly, if the arrow of the kernel in a type-IB component (see figure 15 (b)) is pointing away from the root (i.e. the second term of eq. (3.5)), this component should be dressed by an extra minus. Since the choice of $y \in \mathscr{L} \backslash\{r\}$ in eq. (4.3) does not affect the direction of arrows in the final lower block $\mathscr{L}$, the number of such type-IB components only depends on $\mathscr{U}, \mathscr{L}$ and the choice of $x \in \mathscr{U}$ in eq. (4.3). We use $\operatorname{Tr}\left(\mathscr{U}_{x}, \mathscr{L}\right)$ to denote this number, the resulting sign is then written as $(-1)^{\operatorname{Tr}\left(\mathscr{U}_{x}, \mathscr{L}\right)}$.

(iii) For any graph, the total number $\mathcal{N}\left(\mathscr{U}_{x}\right)+\mathcal{N}(\mathscr{L})+1$ of arrows pointing away from the root induces the third sign $(-1)^{\mathcal{N}\left(\mathscr{U}_{x}\right)+\mathcal{N}(\mathscr{L})+1}$ (as required by the refined graphic rule), where $\mathcal{N}\left(\mathscr{U}_{x}\right)$ and $\mathcal{N}(\mathscr{L})$ count the corresponding numbers in $\mathscr{U}$ (for $x \in \mathscr{U}$ ) and $\mathscr{L}$. The extra minus is caused by the type-3 line between $\mathscr{U}$ and $\mathscr{L}$.

To sum up, the sign for any (physical or spurious) graph is given by

$$
(-)^{\mathcal{G}}=(-1)^{S\left(\mathscr{U}_{x}\right)+\operatorname{Tr}\left(\mathscr{U}_{x}, \mathscr{L}\right)+\mathcal{N}\left(\mathscr{U}_{x}\right)+\mathcal{N}(\mathscr{L})+1} .
$$

For a physical graph $\mathcal{G}$, there is no spurious component, thus $S\left(\mathscr{U}_{x}\right)=0$. For identities induced from single-trace amplitudes, $\operatorname{Tr}\left(\mathscr{U}_{x}, \mathscr{L}\right)$ vanishes and the sign (4.9) turns into the one given in [1]. 


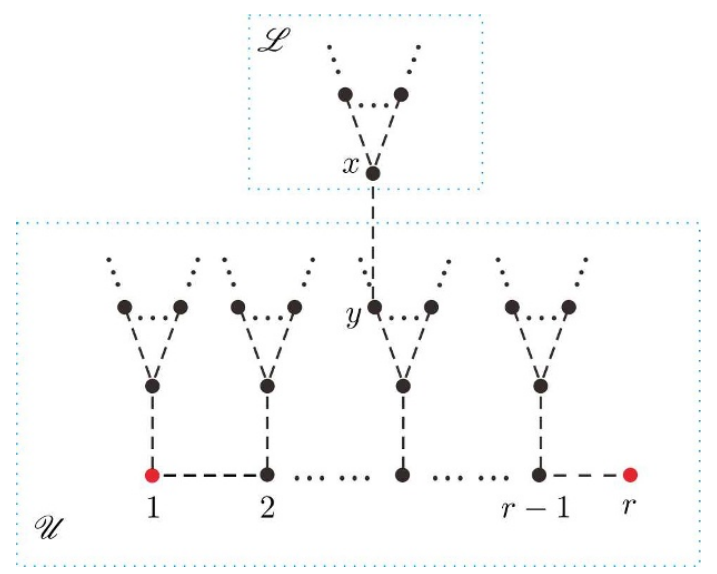

Figure 18. Relative positions of nodes in a graph $\mathcal{G}$ which is determined by $\mathscr{U}, \mathscr{L}$ and $x \in \mathscr{U}$, $y \in \mathscr{L} \backslash\{r\}$ can be characterized by dashed lines with no arrow.

\subsection{Expanding induced identities in terms of BCJ relations}

We are now ready to show the expression in the square brackets in eq. (4.3), i.e.

$$
I[\mathscr{U}, \mathscr{L}] \equiv \sum_{\substack{x \in \mathscr{U} \\ y \in \mathscr{L} \backslash\{r\}}} \sum_{\boldsymbol{\sigma}^{\mathcal{G}}}(-)^{\mathcal{G}}\left(k_{x} \cdot k_{y}\right) A\left(1, \boldsymbol{\sigma}^{\mathcal{G}}, r\right)
$$

for a given configuration of the final upper and lower blocks $\mathscr{U}, \mathscr{L}$ is the l.h.s. of the graphbased BCJ relation (A.13) (hence a combination of traditional BCJ relations (A.12)). Here a graph $\mathcal{G}$ in the above expression is a physical or a spurious graph which is constructed by connecting two nodes $x \in \mathscr{U}, y \in \mathscr{L} \backslash\{r\}$ via a type-3 line. Our discussion is carried out by the follow steps:

(i) According to the refined graphic rule, permutations $\boldsymbol{\sigma}^{\mathcal{G}}$ in eq. (4.10) are independent of the line styles in $\mathcal{G}$. Therefore, all lines in $\mathcal{G}$ can be replaced by dashed lines (with no arrow) which only characterize the relative positions of nodes in $\mathcal{G}$, as shown by figure 18. Apparently, for a given configuration of the final upper and lower blocks $\mathscr{U}, \mathscr{L}$ and a given choice of $x \in \mathscr{L}$, the corresponding permutations $\boldsymbol{\sigma}^{\mathcal{G}}$ satisfy

$$
\left.\boldsymbol{\sigma}^{\mathcal{G}} \in[\zeta \amalg \gamma]\right|_{y \prec x} \quad\left(\text { for }\left.\boldsymbol{\zeta} \in \mathscr{U}\right|_{x} \text { and } \boldsymbol{\gamma} \in\left(\left.\mathscr{L}\right|_{1}\right) \backslash\{1, r\}\right),
$$

where $\left.\mathscr{U}\right|_{x}$ and $\left.\mathscr{L}\right|_{1}$ denote the permutations established by $\mathscr{U}$ and $\mathscr{L}$ when $x \in \mathscr{U}$ and the root $1 \in \mathscr{L}$ are the leftmost elements respectively. Since 1 and $r$ are fixed as the first and the last elements in eq. (4.10), they should be excluded from $\boldsymbol{\sigma}^{\mathcal{G}}$. The $y \prec x$ means $y$ situates before $x$ in the permutation, i.e. $\left(\sigma^{\mathcal{G}}\right)^{-1}(y)<\left(\sigma^{\mathcal{G}}\right)^{-1}(x)$. Noting that the choice of $y$ is independent of the relative orders $\zeta$ and $\gamma$, we rewrite the summations in eq. (4.10) as follows

$$
\sum_{\substack{x \in \mathscr{U} \\ y \in \mathscr{L} \backslash\{r\}}} \sum_{\boldsymbol{\sigma}^{\mathcal{G}}} \rightarrow \sum_{x \in \mathscr{U}} \sum_{\left.\boldsymbol{\zeta} \in \mathscr{U}\right|_{x}} \sum_{\left.\boldsymbol{\gamma} \in \mathscr{L}\right|_{1} \backslash\{1, r\}} \sum_{y \in \mathscr{L} \backslash\{r\}} \sum_{\left.\boldsymbol{\sigma} \in[\boldsymbol{\zeta} \sqcup \boldsymbol{\gamma}]\right|_{y \prec x}} .
$$


(ii) For a given permutation $\boldsymbol{\sigma} \in \boldsymbol{\zeta} \amalg \boldsymbol{\gamma}$, one can collect together the coefficients $k_{x} \cdot k_{y}$ in eq. (4.11) with different choices of $y \in \mathscr{L} \backslash\{r\}$. Specifically, only those $y$ satisfying $y \prec x$ in $\boldsymbol{\sigma}$ have nonzero contributions and the sign (4.9) is totally independent of $y$. Hence, all the $k_{x} \cdot k_{y}$ factors for a given $\boldsymbol{\sigma}$ are collected as $k_{x} \cdot Y_{x}(\boldsymbol{\sigma})$ where $Y_{x}^{\mu}(\boldsymbol{\sigma}) \equiv \sum_{y \prec x} k_{y}^{\mu}$ (the gluon 1 is always included as the leftmost $y$ in this summation). Meanwhile, the last two summations in eq. (4.12) turns into

$$
\sum_{y \in \mathscr{L} \backslash\{r\}} \sum_{\left.\sigma \in[\zeta \uplus \gamma]\right|_{y \prec x}} \rightarrow \sum_{\sigma \in \zeta \amalg \gamma}
$$

(iii) As illustrated by appendix C, the sign $(-)^{\mathcal{G}}$ in eq. (4.10) has the following pattern. Two graphs with $x=x_{1}$ and $x=x_{2}$ where $x_{1}, x_{2} \in \mathscr{U}$ are adjacent to each other must be associated with opposite signs. Hence one can extract the sign eq. (4.9) for a graph with $x=x_{0} \in \mathscr{U}$ as an overall factor and then the sign for an arbitrary choice of node $x$ is given by

$$
(-1)^{S\left(\mathscr{U}_{x_{0}}\right)+\operatorname{Tr}\left(\mathscr{U}_{x_{0}}, \mathscr{L}\right)+\mathcal{N}\left(\mathscr{U}_{x_{0}}\right)+\mathcal{N}(\mathscr{L})+1} f^{x} \equiv(-)^{\mathcal{G}\left(x_{0}\right)} f^{x},
$$

where $f^{x}$ is defined by (i). $f^{x_{0}}=1$, (ii). $f^{x_{1}}=-f^{x_{2}}$ for two adjacent nodes $x_{1}$ and $x_{2}\left(x_{1}, x_{2} \in \mathscr{U}\right)$.

When all the above are taken into account, eq. (4.10) is finally expressed by

$$
I[\mathscr{U}, \mathscr{L}]=(-)^{\mathcal{G}\left(x_{0}\right)} \sum_{\left.\boldsymbol{\gamma} \in \mathscr{L}\right|_{1} \backslash\{1, r\}}\left[\sum_{x \in \mathscr{U}} f^{x} \sum_{\left.\boldsymbol{\zeta} \in \mathscr{U}\right|_{x}} \sum_{\boldsymbol{\sigma} \in \zeta \amalg \boldsymbol{\gamma}}\left(k_{x} \cdot Y_{x}(\boldsymbol{\sigma})\right) A(1, \boldsymbol{\sigma}, r)\right],
$$

where the summation over $\gamma$ was extracted out because it is independent of the choice of $x \in \mathscr{U}$. The expression in the square brackets in eq. (4.15) is nothing but the l.h.s. of the graph-based BCJ relation (A.13).

\section{Conclusions}

In this paper, we provided the refined graphic rule for expanding tree level multi-trace EYM amplitudes in terms of color-ordered YM amplitudes. When the gauge invariance condition of a graviton and the cyclic symmetry of a gluon trace were imposed, this expansion induced two identities (2.19) and (2.21) respectively. By extending the analysis for the singletrace case [1] to an arbitrary multi-trace induced identity, we demonstrated that eq. (2.19) and eq. (2.21) can finally be expressed as a combination of graph-based BCJ relations (thus traditional BCJ relations).

There are several related topics that deserve further study: (i). First, how to understand the induced identities from the view of string theory? String theory studies of the expansions of EYM amplitudes have been established in [6, 9, 33, 34], while BCJ relations have also been proven in string theory $[31,35]$. Hence it is reasonable to expect a stringtheory approach to both induced identities and graph-based BCJ relations. (ii). Second, it is worth investigating the induced identities in various theories systematically. In [36], 
a unified web of expansions of amplitudes was founded with the help of the unifying relation [37], which inspires that the induced identities may exist in many other theories. (iii). Third, the YM expansion of EYM amplitudes, which have been used in this paper, is in KK basis [16]. As pointed in [38], this expansion can be extended to BCJ basis [17]. We expect that the refined graphic rule can also be generalized to expansions in BCJ basis [17]. (iv). Last but not least, a kinematic algebra for constructing BCJ numerators in the MHV sector was proposed [39]. It seems that distinct sectors of numerators are corresponding to graphs with different numbers of type-IA kernels. Thus, one may provide a general rule for constructing all sectors of BCJ numerators, with the help of refined graphic rule.

\section{Acknowledgments}

The authors are grateful to Chih-Hao Fu, Song He, Xiaodi Li, Hui Luo, Gang Yang, Yihong Wang and Yong Zhang for helpful discussions or/and valuable comments. This work is supported by NSFC under Grant Nos. 11875206, 11847309, Jiangsu Ministry of Science and Technology under contract BK20170410 as well as the "Fundamental Research Funds for the Central Universities".

\section{A Recursive expansions and BCJ relations}

In this section, we review the recursive expansions of multi-trace EYM amplitudes and the BCJ relations for YM amplitudes.

\section{A.1 Recursive expansions of multi-trace EYM amplitudes}

When all gravitons $h_{1}, \ldots, h_{s}$ and gluon traces $\mathbf{2}, \ldots, \boldsymbol{m}$ are collected into the set $\mathcal{H} \equiv$ $\left\{h_{1}, \ldots, h_{s}, \mathbf{2}, \ldots, \boldsymbol{m}\right\}$, a tree level multi-trace EYM amplitude $A(1,2, \ldots, r|\mathbf{2}| \ldots \mid \boldsymbol{m} \| \mathbf{H})$ where $\mathbf{H} \equiv\left\{h_{1}, h_{2}, \ldots, h_{s}\right\}$ can be briefly expressed by $A(1,2, \ldots, r \| \mathcal{H})$. As proven in [2], this multi-trace amplitude satisfies the following recursive expansion relation:

$$
A(1,2, \ldots, r \| \mathcal{H})=\sum_{\substack{\mathcal{H} \backslash\left\{\mathcal{H}_{a}\right\} \\ \rightarrow \text { perms } \mathcal{H}_{A} \mid \mathcal{H}_{B}}} \widetilde{\operatorname{Tr}}\left[\sum_{\boldsymbol{\sigma}} C(1, \boldsymbol{\sigma}, r) A\left(1, \boldsymbol{\sigma}, r \| \boldsymbol{H}_{B}\right)\right]
$$

In the expansion (A.1), we have picked out an arbitrary element $\mathcal{H}_{a}$ (a graviton or a gluon trace) from $\mathcal{H}$, which is called the fiducial element. Apparently, $\mathcal{H}_{a}$ can be either a graviton or a gluon trace. On the r.h.s. of eq. (A.1):

- The first summation is taken over (i). all possible splittings of the set $\mathcal{H} \backslash\left\{\mathcal{H}_{a}\right\}$ into two subsets $\mathcal{H}_{A}, \mathcal{H}_{B}$ and (ii). all permutations of elements in $\mathcal{H}_{A}$ for a given splitting. 
- For a given splitting of $\mathcal{H} \backslash\left\{\mathcal{H}_{a}\right\}$ and a given permutation of elements in $\mathcal{H}_{A}$, the summation $\widetilde{\sum}_{\mathrm{Tr}}$ is defined as follows

$$
\begin{aligned}
& \text { if } \mathcal{H}_{a} \text { is a graviton: } \overline{\sum_{\operatorname{Tr}}} \rightarrow \sum_{\substack{\left\{a_{i}, b_{i}\right\} \subset \boldsymbol{t}_{i} \\
\text { for all } \boldsymbol{t}_{i} \in \mathcal{H}_{A}}}(-1)^{\left|\boldsymbol{t}_{i}, a_{i}, b_{i}\right|} \sum_{\boldsymbol{\beta}_{i}} \\
& \text { if } \mathcal{H}_{a}=\boldsymbol{t}_{0} \text { is a gluon trace: } \widetilde{\sum_{\operatorname{Tr}}} \rightarrow \sum_{\substack{a_{0} \in \boldsymbol{t}_{0} \\
\text { for } a_{0} \neq b_{0}\left(b_{0} \in \boldsymbol{t}_{0}\right)}}(-1)^{\left|\boldsymbol{t}_{0}, a_{0}, b_{0}\right|} \\
& \times \sum_{\boldsymbol{\beta}_{0}} \sum_{\substack{\left\{a_{i}, b_{i}\right\} \subset \boldsymbol{t}_{i} \\
\text { for all } \boldsymbol{t}_{i} \in \mathcal{H}_{A}}}(-1)^{\left|\boldsymbol{t}_{i}, a_{i}, b_{i}\right|} \sum_{\boldsymbol{\beta}_{i}} .
\end{aligned}
$$

This means we sum over all possible choices of the ordered pair of gluons $\left\{a_{i}, b_{i}\right\} \subset \boldsymbol{t}_{i}$ for all traces $\boldsymbol{t}_{i} \in \mathcal{H}_{A}$. If the fiducial element $\mathcal{H}_{a}$ is also a trace, namely $\boldsymbol{t}_{0}$, we should fix an arbitrary gluon $b_{0} \in \boldsymbol{t}_{0}$ and then sum over all choices of $a_{0} \neq b_{0}$ in this trace. For a given choice of $a_{i}$ and $b_{i}$, a gluon trace (including $\mathcal{H}_{a}$ if it is also a trace) can always be written into the form $a_{i}, \boldsymbol{X}_{i}, b_{i}, \boldsymbol{Y}_{i}$ where $\boldsymbol{X}_{i}$ and $\boldsymbol{Y}_{i}$ are the two ordered sets of gluons separated by $a_{i}$ and $b_{i}$. The sign $(-1)^{\left|\boldsymbol{t}_{i}, a_{i}, b_{i}\right|}$ in eq. (A.2) and eq. (A.3) for each trace is defined as $(-1)^{\left|\boldsymbol{Y}_{i}\right|}$ where $\left|\boldsymbol{Y}_{i}\right|$ is the number of elements in $\boldsymbol{Y}_{i}$. Permutations $\boldsymbol{\beta}_{i}$ (for a given $\left\{a_{i}, b_{i}\right\}$ ) and $\boldsymbol{\beta}_{0}$ (for a given $a_{0} \neq b_{0}$ ) in eq. (A.2) and/or eq. (A.3) which satisfy

$$
\begin{aligned}
& \boldsymbol{\beta}_{i} \in \mathrm{KK}\left[\boldsymbol{t}_{i}, a_{i}, b_{i}\right] \equiv \boldsymbol{X}_{i} ш \boldsymbol{Y}_{i}^{T}, \\
& \boldsymbol{\beta}_{0} \in \mathrm{KK}\left[\boldsymbol{t}_{0}, a_{0}, b_{0}\right] \equiv \boldsymbol{X}_{0} ш \boldsymbol{Y}_{0}^{T},
\end{aligned}
$$

are also summed over.

- Supposing that the permutation of elements in $\mathcal{H}_{A}$ in the first summation (see eq. (A.1)) is given by $j_{1}, j_{2}, \ldots, j_{u}$ and the gluon pairs in the second summation are $\left\{a_{i}, b_{i}\right\} \subset \boldsymbol{t}_{i}\left(i\right.$ can be 0 if the fiducial element $\mathcal{H}_{a}$ is a gluon trace $\left.\boldsymbol{t}_{0}\right)$, we sum over all permutations $\sigma$ satisfying

$$
\boldsymbol{\sigma} \in\{2, \ldots, r-1\} \amalg\left\{j_{1}, j_{2}, \ldots, j_{u}, \mathcal{H}_{a}\right\} .
$$

Here the traces in the ordered set $\left\{j_{1}, j_{2}, \ldots, j_{u}, \mathcal{H}_{a}\right\}$ are no longer considered as single elements but considered as proper permutations of all gluons in them. Particularly, if $j_{i}$ denotes a gluon trace $\boldsymbol{t}_{i}$ and this trace can be written as $a_{i}, \boldsymbol{X}_{i}, b_{i}, \boldsymbol{Y}_{i}$ for a given choice of ordered pair $\left\{a_{i}, b_{i}\right\} \subset \boldsymbol{t}_{i}$, we should replace $j_{i}$ in eq. (A.6) by a permutation $\left\{a_{i}, \boldsymbol{\beta}_{i}, b_{i}\right\}$ where $\boldsymbol{\beta}_{i}$ satisfies eq. (A.4). Similarly, if the fiducial element $\mathcal{H}_{a}$ is also a trace, say $\boldsymbol{t}_{0}$, it must be replaced by a permutation $\left\{a_{0}, \boldsymbol{\beta}_{0}, b_{0}\right\}$ where $\boldsymbol{\beta}_{0}$ satisfies eq. (A.5). Then the summation over $\sigma$ in eq. (A.1) means summing over all possible shuffle permutations in eq. (A.6).

- The coefficient $C(1, \boldsymbol{\sigma}, r)$ in eq. (A.1) is defined as

$$
\mathcal{E}_{\mathcal{H}_{a}} \cdot \mathbb{F}_{j_{u}} \cdot \ldots \cdot \mathbb{F}_{j_{i}} \cdot Y_{j_{1}}(\boldsymbol{\sigma}),
$$


where $\mathcal{E}^{\mu}$ and $\mathbb{F}^{\mu \nu}$ are defined by eq. (2.7) and eq. (2.8).

$$
Y_{j_{1}}^{\mu}(\boldsymbol{\sigma})=\left\{\begin{array}{ll}
\sum_{\sigma^{-1}(l)<\sigma^{-1}\left(h_{x}\right)} k_{l}^{\mu} & \left(\text { if } j_{1} \text { is a graviton } h_{x}\right) \\
\sum_{\sigma^{-1}(l)<\sigma^{-1}\left(b_{i}\right)} k_{l}^{\mu} & \left(\text { if } j_{1} \text { is a gluon trace } \boldsymbol{t}_{i}\right)
\end{array} .\right.
$$

\section{A.2 Identities induced from multi-trace EYM amplitudes}

Symmetries of multi-trace EYM amplitudes, together with the recursive expansion (A.1), induce nontrivial identities for EYM amplitudes with fewer gravitons and/or gluon traces [2]. There are two symmetries under consideration in this paper: the gauge invariance condition for a graviton and the cyclic symmetry of a gluon trace.

Muti-trace EYM amplitude satisfies gauge invariance condition, which states that the amplitude has to vanish once half polarization $\epsilon_{h_{x}}^{\mu}$ of a graviton $h_{x}$ is replaced by the momentum $k_{h_{x}}^{\mu}$. If this replacement is performed on the r.h.s. of the recursive expansion (A.1), we should consider two distinct situations:

- If the fiducial element $\mathcal{H}_{a}$ is a graviton $h_{x}$ (i.e. eq. (A.1) is type-I expansion), the gauge invariance condition for $h_{x}$ induces a nontrivial relation between EYM amplitudes with fewer gravitons

$$
\sum_{\substack{\mathcal{H} \backslash h_{x} \\ \rightarrow \operatorname{perms} \mathcal{H}_{A} \mid \mathcal{H}_{B}}} \widetilde{\operatorname{Tr}}\left[\left.\sum_{\boldsymbol{\sigma}} C(1, \boldsymbol{\sigma}, r)\right|_{\epsilon_{h_{x}} \rightarrow k_{h_{x}}} A\left(1, \boldsymbol{\sigma}, r \| \mathcal{H}_{B}\right)\right]=0 .
$$

- If $h_{x}$ is not the fiducial one, it may belong to either $\mathcal{H}_{A}$ or $\boldsymbol{\mathcal { H }}_{B}$ in eq. (A.1) . Terms with $h_{x} \in \mathcal{H}_{A}$ have to vanish due to the antisymmetry of the strength tensor $F_{h_{x}}^{\mu \nu} \equiv$ $k_{h_{x}}^{\mu} \epsilon_{h_{x}}^{\nu}-k_{h_{x}}^{\nu} \epsilon_{h_{x}}^{\mu}$, while terms with $h_{x} \in \mathcal{H}_{B}$ have to vanish due to gauge invariance of amplitudes with fewer gravitons.

Therefore, the only nontrivial identity induced by the gauge invariance condition of a graviton is eq. (A.9) which is called type-I identity in [2].

Another identity (called type-II identity in [2]) is induced from the expansion (A.1) where the fiducial element $\mathcal{H}_{a}$ is a gluon trace $\boldsymbol{t}_{0}$. In particular, we notice that the end element $b_{0} \in \boldsymbol{t}_{0}$ can be chosen arbitrarily in the fiducial trace $\boldsymbol{t}_{0}$. This arbitrariness is essentially caused by the cyclic symmetry of the trace $t_{0}[2]$ and indicates the following identity

$$
\sum_{\substack{\mathcal{H} \backslash \mathcal{H}_{a} \\ \rightarrow \text { perms } \mathcal{H}_{A} \mid \mathcal{H}_{B}}} \widetilde{\sum_{\operatorname{Tr}}}\left[\sum_{\boldsymbol{\sigma}^{\prime}} C\left(1, \boldsymbol{\sigma}^{\prime}, r\right) A\left(1, \boldsymbol{\sigma}^{\prime}, r \| \mathcal{H}_{B}\right)\right]=0,
$$

where $\boldsymbol{\sigma}^{\prime}$ is defined by

$$
\boldsymbol{\sigma}^{\prime} \in\{2, \ldots, r-1\} \amalg\left\{j_{1}, j_{2}, \ldots, j_{u}, a_{0}, \boldsymbol{\beta}_{0} \in \mathrm{KK}\left[\boldsymbol{t}_{0}, a_{0}, b_{0}\right], \varnothing_{Q}\right\} .
$$


Here we removed $b_{0}$ from the trace $\boldsymbol{t}_{0}$ first, then shuffled these permutations according to eq. (A.6). The $j_{1}, j_{2}, \ldots, j_{u}$ is a permutation of elements in $\mathcal{H}_{A}$.

When the recursive expansion (A.1) for amplitudes with fewer gravitons and/or gluon traces are applied repeatedly, the two types of relations (A.9) and (A.10) respectively turn into the induced identities (2.19) and (2.21) for pure YM amplitudes.

\section{A.3 BCJ relations}

Tree level color-ordered YM amplitudes satisfy the following traditional BCJ relation $[31,32]$ :

$$
\sum_{\boldsymbol{\sigma} \in \boldsymbol{\beta} \amalg \boldsymbol{\alpha}} \sum_{l \in \boldsymbol{\beta}}\left(k_{l} \cdot X_{l}(\boldsymbol{\sigma})\right) A(1, \boldsymbol{\sigma}, r)=0,
$$

where $\beta$ and $\boldsymbol{\alpha}$ are two ordered sets of external gluons, $X_{l}(\boldsymbol{\sigma})$ denotes the sum of all momenta of gluons $a \in\{1, \boldsymbol{\sigma}\} \cup \beta$ satisfying $\sigma^{-1}(a)<\sigma^{-1}(l)$.

In [1], the following graph-based BCJ relation for YM amplitudes was proposed

$$
\sum_{a \in \mathcal{T}} f^{a} \sum_{\left.\boldsymbol{\zeta} \in \mathcal{T}\right|_{a}} \sum_{\boldsymbol{\sigma} \in \boldsymbol{\zeta} \uplus \boldsymbol{\gamma}}\left[k_{a} \cdot Y_{a}(\boldsymbol{\sigma})\right] A(1, \boldsymbol{\sigma}, r)=0 .
$$

Here, $\gamma$ is an arbitrary permutation of elements in $\{2, \ldots, r-1\}$ and $\mathcal{T}$ is an arbitrary connected tree graph. When a node $a$ is chosen as the leftmost element, the tree graph $\mathcal{T}$ establishes permutations $\left.\zeta \in \mathcal{T}\right|_{a}$ as follows (i). For two adjacent nodes $x$ and $y$, if $x$ is nearer to $a$ than $y$, we have $\zeta^{-1}(x)<\zeta^{-1}(y)$, (ii). If there are subtree structures attached to a same node, we should shuffle the permutations established by these subtrees together. The factor $f^{a}$ is a relative sign depending on the node $a$. This factor is determined by the following steps. (i). Choose an arbitrary node $c$ and require $f^{c}=1$. (ii). For arbitrary two adjacent nodes $c_{1}$ and $c_{2}$, we have $f^{c_{1}}=-f^{c_{2}}$. As already proven in [1], the graph-based BCJ relation (A.13) can always be written as a combination of the traditional ones (A.12).

\section{B Proof of eq. (3.5)}

To prove eq. (3.5), we focus on a term with a given choice of $\left\{a_{i}, b_{i}\right\} \subset \boldsymbol{i}$ in eq. (3.5) and prove the following stronger relation:

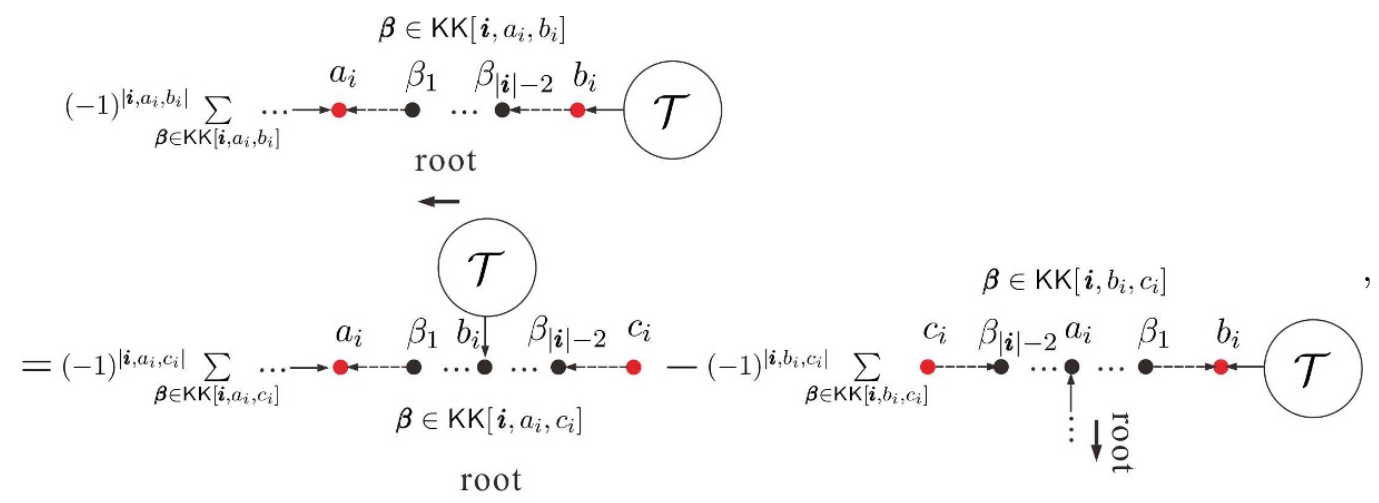


where the $\mathcal{T}$ denotes a tree structure which is attached to the node $b_{i}$. In fact, all these nodes in the trace can be attached by arbitrary tree structures and the relation (B.1) still holds. Once all $\left\{a_{i}, b_{i}\right\} \subset \boldsymbol{i}$ are summed over, we arrive the relation (3.5).

Suppose the gluons in trace $\boldsymbol{i}$ are in the cyclic order $d_{1}, d_{2}, \ldots, d_{|\boldsymbol{i}|}$. Without loss of generality, $a_{i}$ and $b_{i}$ are respectively chosen as $a_{i}=d_{j}, b_{i}=d_{|\boldsymbol{i}|}$. Then the permutations $\left\{a_{i}, \boldsymbol{\beta} \in \mathrm{KK}\left[\boldsymbol{i}, a_{i}, b_{i}\right], b_{i}\right\}$ on the 1.h.s. of eq. (B.1) are explicitly displayed by

$$
\begin{aligned}
& \left\{a_{i}=d_{j},\left\{d_{j+1}, \ldots, d_{|\boldsymbol{i}|-1}\right\} \amalg\left\{d_{j-1}, \ldots, d_{1}\right\}, b_{i}=d_{|\boldsymbol{i}|},\left.\mathcal{T}\right|_{x}\right\}, \\
& =\left.\left\{\left.\left\{a_{i}=d_{j},\left\{d_{j+1}, \ldots, d_{|\boldsymbol{i}|-1}\right\} \uplus\left\{d_{j-1}, \ldots, d_{1}\right\}, b_{i}=d_{|\boldsymbol{i}|}\right\} \amalg \mathcal{T}\right|_{x}\right\}\right|_{b_{i} \prec x} .
\end{aligned}
$$

with a sign $(-1)^{\left|\boldsymbol{i}, a_{i}, b_{i}\right|}=(-1)^{j-1}$ (the overall sign coming from $\left.\mathcal{T}\right|_{x}$ has been neglected). Here $x$ denotes the nearest to $b_{i}$ node in $\mathcal{T}$ and $\left.\mathcal{T}\right|_{x}$ are the permutations established by the tree $\mathcal{T}$. The notation $b_{i} \prec x$ means the position of $b_{i}$ in the permutation is less than that of $x$. The second line of eq. (B.2), where we shuffled $\left.\mathcal{T}\right|_{x}$ with the full trace and required the node $b_{i}$ is always on the left of the node $x$, is apparently equivalent to the first line.

On the r.h.s. of eq. (B.1), we assume that the $c_{i}$ is chosen as $d_{l}(l>j)$. The case with $l<j$ follows from a similar discussion. Then permutations in the first term on the r.h.s. of eq. (B.1) is given by

$$
\left.\left\{\left.\left\{a_{i}=d_{j},\left\{d_{j+1}, \ldots, d_{l-1}\right\} \uplus\left\{d_{j-1}, \ldots, d_{1}, b_{i}=d_{|i|}, \ldots, d_{l+1}\right\}, c_{i}=d_{l}\right\} ш \mathcal{T}\right|_{x}\right\}\right|_{b_{i} \prec x},
$$

with the sign $(-1)^{\left|i, a_{i}, c_{i}\right|}=(-1)^{|\boldsymbol{i}|-l+j-1}$. The permutations in the second term of the r.h.s. of eq. (3.5) are given by the following two steps:

- First shuffle the gluons in the trace $i$ so that $b_{i}=d_{|i|}$ and $c_{i}=d_{l}$ become the two ends of the trace. Then the permutations $\beta \in \mathrm{KK}\left[\boldsymbol{i}, b_{i}, c_{i}\right]$ are explicitly given by

$$
\beta \in\left\{d_{|i|-1}, \ldots, d_{l+1}\right\} ш\left\{d_{1}, \ldots, d_{l-1}\right\} .
$$

which is associated with a sign $(-1)^{\left|\boldsymbol{i}, b_{i}, c_{i}\right|}=(-1)^{|\boldsymbol{i}|-l-1}$. These permutations can be classified according to the relative orders between the $a_{i}=d_{j}\left(d_{j} \in\left\{d_{1}, \ldots, d_{l-1}\right\}\right)$ and elements in the set $\left\{d_{|i|-1}, \ldots, d_{l+1}\right\}$ :

$$
\begin{aligned}
& \boldsymbol{\beta}^{(1)} \in\left\{d_{1}, \ldots, d_{j-1}, d_{j},\left\{d_{j+1}, \ldots, d_{l-1}\right\} \amalg\left\{d_{|\boldsymbol{i}|-1}, \ldots, d_{l+1}\right\}\right\} \\
& \boldsymbol{\beta}^{(2)} \in\left\{\left\{d_{1}, \ldots, d_{j-1}\right\} \amalg\left\{d_{|\boldsymbol{i}|-1}\right\}, d_{j},\left\{d_{j+1}, \ldots, d_{l-1}\right\} \amalg\left\{d_{|\boldsymbol{i}|-2}, \ldots, d_{l+1}\right\}\right\} \\
& \text {... } \\
& \beta^{(q)} \in\left\{\left\{d_{1}, \ldots, d_{j-1}\right\} \amalg\left\{d_{|i|-1}, \ldots, d_{|i|-q+1}\right\},\right. \\
& \left.d_{j},\left\{d_{j+1}, \ldots, d_{l-1}\right\} \uplus\left\{d_{|\boldsymbol{i}|-q}, \ldots, d_{l+1}\right\}\right\} \\
& \boldsymbol{\beta}^{(|\boldsymbol{i}|-l-1)} \in\left\{\left\{d_{1}, \ldots, d_{j-1}\right\} \uplus\left\{d_{|\boldsymbol{i}|-1}, \ldots, d_{l+2}\right\}, d_{j},\left\{d_{j+1}, \ldots, d_{l-1}\right\} \amalg\left\{d_{l+1}\right\}\right\} \\
& \boldsymbol{\beta}^{(|\boldsymbol{i}|-l)} \in\left\{\left\{d_{1}, \ldots, d_{j-1}\right\} \amalg\left\{d_{|\boldsymbol{i}|-1}, \ldots, d_{l+1}\right\}, d_{j}, d_{j+1}, \ldots, d_{l-1}\right\} .
\end{aligned}
$$

- For any given permutation $\boldsymbol{\beta}^{(q)}$ in eq. (B.5), the permutations $\boldsymbol{\sigma}^{(q)}$ established by the second term on the r.h.s. of eq. (B.1) are obtained by shuffling the two branches 
attached to the node $a_{i}=d_{i}$ together:

$$
\begin{aligned}
& \boldsymbol{\sigma}^{(q)} \in\left\{\left\{a_{i}=d_{j},\left\{\left\{d_{j+1}, \ldots, d_{l-1}\right\} \uplus\left\{d_{|i|-q}, \ldots, d_{l+1}\right\}, c_{i}=d_{l}\right\}\right.\right. \\
& \left.\left.\varpi\left\{\left\{d_{j-1}, \ldots, d_{1}\right\} \uplus\left\{d_{|i|-q+1}, \ldots, d_{|i|-1}\right\}, b_{i}=d_{|i|}\right\}\right\}\left.\amalg \mathcal{T}\right|_{x}\right\}\left.\right|_{b_{i} \prec x}
\end{aligned}
$$

with a sign $(-1)^{|\boldsymbol{i}|-l-1}(-1)^{\mathcal{N}_{a_{i}}^{(q)}}$. Here $\mathcal{N}_{a_{i}}^{(q)}$ denotes the number of arrows pointing away from root in the trace $\boldsymbol{i}$.

According to the relative orders between $d_{|\boldsymbol{i}|-q}$ and $d_{|\boldsymbol{i}|-q+1}$, permutations $\boldsymbol{\sigma}^{(q)}$ in eq. (B.7) splits into $\left.\boldsymbol{\sigma}_{\mathrm{A}}^{(q)} \equiv \boldsymbol{\sigma}^{(q)}\right|_{d_{|\boldsymbol{i}|-q} \prec d_{|\boldsymbol{i}|-q+1}}$ and $\left.\boldsymbol{\sigma}_{\mathrm{B}}^{(q)} \equiv \boldsymbol{\sigma}^{(q)}\right|_{d_{|\boldsymbol{i}|-q} \succ d_{|\boldsymbol{i}|-q+1}}$. It is easy to see

$$
\boldsymbol{\sigma}_{\mathrm{A}}^{(q)}=\boldsymbol{\sigma}_{\mathrm{B}}^{(q+1)}, \quad \mathcal{N}_{a_{i}}^{(q)}=\mathcal{N}_{a_{i}}^{(q+1)}+1
$$

Hence the contributions from $\boldsymbol{\sigma}_{\mathrm{A}}^{(q)}$ and $\boldsymbol{\sigma}_{\mathrm{B}}^{(q+1)}$ for all $q=1, \ldots,|\boldsymbol{i}|-l-1$ must cancel one another. The remaining nonzero terms are the two boundaries

$$
\begin{aligned}
& \boldsymbol{\sigma}_{\mathrm{B}}^{(1)} \in\left\{\left\{a_{i}=d_{j},\left\{\left\{d_{j+1}, \ldots, d_{l-1}\right\} ш\left\{d_{|\boldsymbol{i}|-1}, \ldots, d_{l+1}\right\}, c_{i}=d_{l}\right\}\right.\right. \\
& \left.\left.\uplus\left\{d_{j-1}, \ldots, d_{1}, b=d_{|i|}\right\}\right\}\left.\left.\right|_{d_{|i|-1} \succ d_{|i|}} w \mathcal{T}\right|_{x}\right\}\left.\right|_{b_{i} \prec x} \\
& =\left\{\left\{a_{i}=d_{j},\left\{d_{j+1}, \ldots, d_{l-1}\right\}\right.\right. \\
& \left.\left.\uplus\left\{d_{j-1}, \ldots, d_{1}, b_{i}=d_{|\boldsymbol{i}|}, d_{|\boldsymbol{i}|-1}, \ldots, d_{l+1}\right\}, c_{i}=d_{l}\right\}\left.\uplus \mathcal{T}\right|_{x}\right\}\left.\right|_{b_{i} \prec x} . \\
& \boldsymbol{\sigma}_{\mathrm{A}}^{(|\boldsymbol{i}|-l)} \in\left\{\left\{a_{i}=d_{j},\left\{d_{j+1}, \ldots, d_{l-1}, c_{i}=d_{l}\right\}\right.\right. \\
& \left.\left.\varpi\left\{\left\{d_{j-1}, \ldots, d_{1}\right\} \amalg\left\{d_{l+1}, \ldots, d_{|i|-1}\right\}, b_{i}=d_{|i|}\right\}\right\}\left.\left.\right|_{d_{l} \prec d_{l+1}} \uplus \mathcal{T}\right|_{x}\right\}\left.\right|_{b_{i} \prec x} \\
& =\left.\left\{\left.\left\{a_{i}=d_{j},\left\{d_{j-1}, \ldots, d_{1}\right\} ш\left\{d_{j+1}, \ldots, d_{|i|-1}\right\}, b_{i}=d_{|i|}\right\} \uplus \mathcal{T}\right|_{x}\right\}\right|_{b_{i} \prec x},
\end{aligned}
$$

with the signs $(-1)^{|\boldsymbol{i}|-l-1}(-1)^{\mathcal{N}_{a_{i}}^{(1)}}=(-1)^{|\boldsymbol{i}|-l-1+j}$ and $(-1)^{|\boldsymbol{i}|-l-1}(-1)^{\mathcal{N}_{a_{i}}^{(|\boldsymbol{i}|-l)}}=(-1)^{j}$ respectively. An extra minus which is introduced from the second term of eq. (B.1) must also be taken into account. Hence the permutations $\sigma_{\mathrm{B}}^{(1)}$ are same with the permutations in eq. (B.3), with an opposite sign. As a result, they cancel with each other. The remaining permutations $\boldsymbol{\sigma}_{\mathrm{A}}^{(|i|-l)}$ are nothing but the permutations (B.2) with the corrected sign. Therefore, we have proven the relation (B.1) for a given $\left\{a_{i}, b_{i}\right\}$. After summing over all possible choices of the $\left\{a_{i}, b_{i}\right\}$ pairs, the proof of eq. (3.5) is completed.

Comments on the proof: in the above proof, the tree structure $\left.\mathcal{T}\right|_{x}$ is always treated separately from the trace in each step (i.e. it is shuffled with the full trace with a proper constraint, as shown in eqs. (B.2), (B.3), (B.7), (B.8)). Thus manipulations on nodes inside the trace are independent of $\left.\mathcal{T}\right|_{x}$. This observation allows us to generalize eq. (3.5) to cases where more tree structures are attached to nodes in the trace $\boldsymbol{i}$ straightforwardly. 


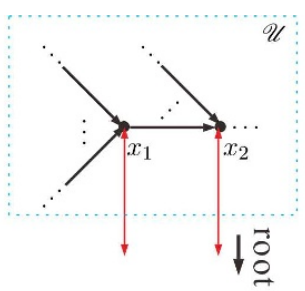

(a)

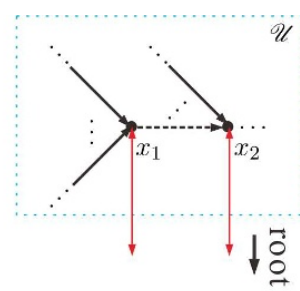

(b)

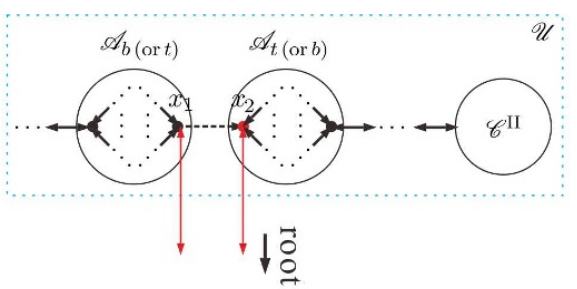

(c)

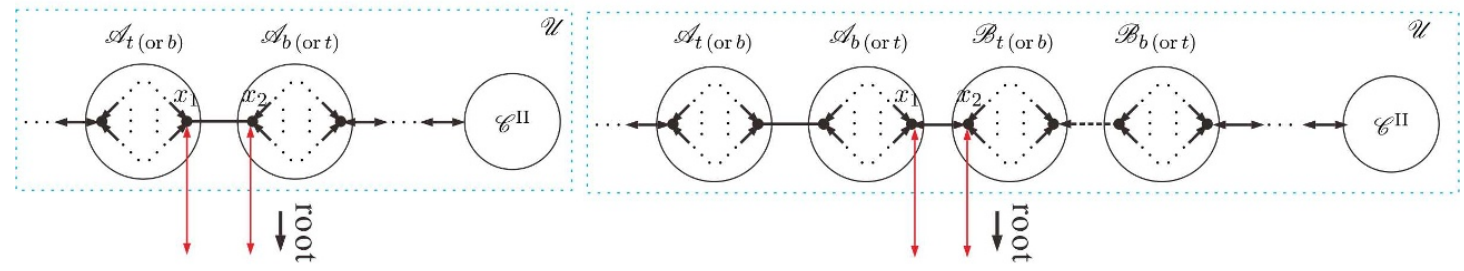

(d)

(e)

Figure 19. All possible situations when choosing $x \in \mathscr{U}$ as two adjacent nodes $x_{1}$ and $x_{2}$.

\section{The sign in eq. (4.10)}

In eq. (4.10), the graph $\mathcal{G}$ is constructed by connecting $x \in \mathscr{U}$ and $y \in \mathscr{L}$ via a type-3 line. As mentioned before, the sign for such a graph is dependent of the choice of $x$ but independent of the choice of $y$. Now we show that two graphs with choosing adjacent $x \in \mathscr{U}$ must have opposite signs. To see this, we study all possible structures presented by figure 19 (a)-(e), where $x=x_{1}$ is adjacent to $x=x_{2}\left(x_{1}, x_{2} \in \mathscr{U}\right)$, as follows.

(i) As shown by figure 19 (a), nodes $x_{1}$ and $x_{2}$ connected by a type-3 line must belong to a same (top or bottom) side of a type-IA or type-IB component. In this case, each one of $S\left(\mathscr{U}_{x}\right), \operatorname{Tr}\left(\mathscr{U}_{x}, \mathscr{L}\right)$ and $\mathcal{N}(\mathscr{L})$ is the same for choosing two adjacent $x$ 's. But the numbers $\mathcal{N}\left(\mathscr{U}_{x}\right)$ in eq. (4.9) for $x=x_{1}$ and $x=x_{2}$ differ by one because the type-2 line between $x_{1}$ and $x_{2}$ has opposite directions for these two cases.

(ii) If $x_{1}$ and $x_{2}$ are connected by a type- 4 line, they must belong to a same typeIB component. If the type- 4 line is not the kernel of the type-IB component, the structure is given by figure 19 (b). In this case, $x_{1}$ and $x_{2}$ must belong to a same side of the component. The numbers $\mathcal{N}\left(\mathscr{U}_{x}\right)$ for choosing $x=x_{1}$ and $x=x_{2}$ should differ by one because the type- 4 line between $x_{1}$ and $x_{2}$ has opposite directions. Each of $S\left(\mathscr{U}_{x}\right), \operatorname{Tr}\left(\mathscr{U}_{x}, \mathscr{L}\right)$ and $\mathcal{N}(\mathscr{L})$ in eq. (4.9) is the same for both choices of $x$.

(iii) If the two adjacent nodes $x_{1}$ and $x_{2}$ connected by a type- 4 line belong to opposite sides of a type-IB component, the type- 4 line must be the kernel of the type-IB component (as shown by figure 19 (c)). In this case, each of $\mathcal{N}\left(\mathscr{U}_{x}\right), \operatorname{Tr}\left(\mathscr{U}_{x}, \mathscr{L}\right)$ and $S\left(\mathscr{U}_{x}\right)$ for $x=x_{1}$ and $x=x_{2}$ differ by one but $\mathcal{N}(\mathscr{L})$ is the same.

(iv) If $x_{1}$ and $x_{2}$ are connected by a type-1 line which must be the kernel of a type-IA component (as shown by figure $19(\mathrm{~d})$ ), only the number $S\left(\mathscr{U}_{x}\right)$ for choosing $x=x_{1}$ 
and $x=x_{2}$ differ by one. Any of $\operatorname{Tr}\left(\mathscr{U}_{x}, \mathscr{L}\right), \mathcal{N}\left(\mathscr{U}_{x}\right)$ and $\mathcal{N}(\mathscr{L})$ is the same for the two choices of $x$.

(v) If $x_{1}$ and $x_{2}$ are connected by a type-3 line as shown by figure 19 (e), only $S\left(\mathscr{U}_{x}\right)$ for choosing $x=x_{1}$ and $x=x_{2}$ differ by one. Any other number is the same for the two choices.

Therefore, in all the above cases, $S\left(\mathscr{U}_{x}\right)+\operatorname{Tr}\left(\mathscr{U}_{x}, \mathscr{L}\right)+\mathcal{N}\left(\mathscr{U}_{x}\right)+\mathcal{N}(\mathscr{L})+1$ for choosing $x=x_{1}$ and $x=x_{2}$, where $x_{1}, x_{2} \in \mathscr{U}$ are two adjacent nodes, must differ by an odd number. In other words, two graphs with adjacent $x$ 's have opposite signs.

Open Access. This article is distributed under the terms of the Creative Commons Attribution License (CC-BY 4.0), which permits any use, distribution and reproduction in any medium, provided the original author(s) and source are credited.

\section{References}

[1] L. Hou and Y.-J. Du, A graphic approach to gauge invariance induced identity, JHEP 05 (2019) 012 [arXiv: 1811.12653] [INSPIRE].

[2] Y.-J. Du, B. Feng and F. Teng, Expansion of all multitrace tree level EYM amplitudes, JHEP 12 (2017) 038 [arXiv: 1708.04514] [INSPIRE].

[3] C.-H. Fu, Y.-J. Du, R. Huang and B. Feng, Expansion of Einstein-Yang-Mills amplitude, JHEP 09 (2017) 021 [arXiv: 1702.08158] [INSPIRE].

[4] M. Chiodaroli, M. Günaydin, H. Johansson and R. Roiban, Explicit formulae for Yang-Mills-Einstein amplitudes from the double copy, JHEP 07 (2017) 002 [arXiv:1703.00421] [INSPIRE].

[5] F. Teng and B. Feng, Expanding Einstein-Yang-Mills by Yang-Mills in CHY frame, JHEP 05 (2017) 075 [arXiv: 1703.01269] [INSPIRE].

[6] S. Stieberger and T.R. Taylor, New relations for Einstein-Yang-Mills amplitudes, Nucl. Phys. B 913 (2016) 151 [arXiv:1606.09616] [InSPIRE].

[7] D. Nandan, J. Plefka, O. Schlotterer and C. Wen, Einstein-Yang-Mills from pure Yang-Mills amplitudes, JHEP 10 (2016) 070 [arXiv: 1607.05701] [INSPIRE].

[8] L. de la Cruz, A. Kniss and S. Weinzierl, Relations for Einstein-Yang-Mills amplitudes from the CHY representation, Phys. Lett. B 767 (2017) 86 [arXiv:1607.06036] [INSPIRE].

[9] O. Schlotterer, Amplitude relations in heterotic string theory and Einstein-Yang-Mills, JHEP 11 (2016) 074 [arXiv: 1608.00130] [inSPIRE].

[10] R. Britto, F. Cachazo and B. Feng, New recursion relations for tree amplitudes of gluons, Nucl. Phys. B 715 (2005) 499 [hep-th/0412308] [INSPIRE].

[11] R. Britto, F. Cachazo, B. Feng and E. Witten, Direct proof of tree-level recursion relation in Yang-Mills theory, Phys. Rev. Lett. 94 (2005) 181602 [hep-th/0501052] [INSPIRE].

[12] Y.-J. Du and Y. Zhang, Gauge invariance induced relations and the equivalence between distinct approaches to NLSM amplitudes, JHEP 07 (2018) 177 [arXiv:1803.01701] [INSPIRE]. 
[13] Y.-J. Du and C.-H. Fu, Explicit BCJ numerators of nonlinear simga model, JHEP 09 (2016) 174 [arXiv: 1606.05846] [INSPIRE].

[14] J.J.M. Carrasco, C.R. Mafra and O. Schlotterer, Abelian Z-theory: NLSM amplitudes and $\alpha^{\prime}$-corrections from the open string, JHEP 06 (2017) 093 [arXiv: 1608.02569] [INSPIRE].

[15] Y.-J. Du and F. Teng, BCJ numerators from reduced Pfaffian, JHEP 04 (2017) 033 [arXiv: 1703.05717] [INSPIRE].

[16] R. Kleiss and H. Kuijf, Multi-gluon cross-sections and five jet production at hadron colliders, Nucl. Phys. B 312 (1989) 616 [inSPIRE].

[17] Z. Bern, J.J.M. Carrasco and H. Johansson, New relations for gauge-theory amplitudes, Phys. Rev. D 78 (2008) 085011 [arXiv:0805.3993] [INSPIRE].

[18] N. Arkani-Hamed, L. Rodina and J. Trnka, Locality and unitarity of scattering amplitudes from singularities and gauge invariance, Phys. Rev. Lett. 120 (2018) 231602 [arXiv: 1612.02797] [INSPIRE].

[19] F. Cachazo, S. He and E.Y. Yuan, Scattering equations and Kawai-Lewellen-Tye orthogonality, Phys. Rev. D 90 (2014) 065001 [arXiv:1306.6575] [InSPIRE].

[20] F. Cachazo, S. He and E.Y. Yuan, Scattering of massless particles in arbitrary dimensions, Phys. Rev. Lett. 113 (2014) 171601 [arXiv:1307.2199] [inSPIRE].

[21] F. Cachazo, S. He and E.Y. Yuan, Scattering of massless particles: scalars, gluons and gravitons, JHEP 07 (2014) 033 [arXiv:1309.0885] [INSPIRE].

[22] F. Cachazo, S. He and E.Y. Yuan, Scattering equations and matrices: from Einstein to Yang-Mills, DBI and NLSM, JHEP 07 (2015) 149 [arXiv:1412.3479] [INSPIRE].

[23] C.S. Lam and Y.-P. Yao, Role of Möbius constants and scattering functions in Cachazo-He-Yuan scalar amplitudes, Phys. Rev. D 93 (2016) 105004 [arXiv:1512.05387] [INSPIRE].

[24] C.S. Lam and Y.-P. Yao, Evaluation of the Cachazo-He-Yuan gauge amplitude, Phys. Rev. D 93 (2016) 105008 [arXiv: 1602.06419] [INSPIRE].

[25] N.E.J. Bjerrum-Bohr, J.L. Bourjaily, P.H. Damgaard and B. Feng, Analytic representations of Yang-Mills amplitudes, Nucl. Phys. B 913 (2016) 964 [arXiv:1605.06501] [InSPIRE].

[26] R. Huang, Y.-J. Du and B. Feng, Understanding the cancelation of double poles in the Pfaffian of CHY-formulism, JHEP 06 (2017) 133 [arXiv: 1702.05840] [INSPIRE].

[27] X. Gao, S. He and Y. Zhang, Labelled tree graphs, Feynman diagrams and disk integrals, JHEP 11 (2017) 144 [arXiv: 1708.08701] [INSPIRE].

[28] S. He, G. Yan, C. Zhang and Y. Zhang, Scattering forms, worldsheet forms and amplitudes from subspaces, JHEP 08 (2018) 040 [arXiv: 1803.11302] [INSPIRE].

[29] C.S. Lam, Pfaffian diagrams for gluon tree amplitudes, Phys. Rev. D 98 (2018) 076002 [arXiv: 1808.07575] [INSPIRE].

[30] C.S. Lam, Off-shell Yang-Mills amplitude in the Cachazo-He-Yuan formalism, Phys. Rev. D 100 (2019) 045009 [arXiv: 1905.05101] [INSPIRE].

[31] N.E.J. Bjerrum-Bohr, P.H. Damgaard and P. Vanhove, Minimal basis for gauge theory amplitudes, Phys. Rev. Lett. 103 (2009) 161602 [arXiv:0907.1425] [INSPIRE]. 
[32] Y.-X. Chen, Y.-J. Du and B. Feng, A proof of the explicit minimal-basis expansion of tree amplitudes in gauge field theory, JHEP 02 (2011) 112 [arXiv:1101.0009] [INSPIRE].

[33] S. He, F. Teng and Y. Zhang, String amplitudes from field-theory amplitudes and vice versa, Phys. Rev. Lett. 122 (2019) 211603 [arXiv:1812.03369] [INSPIRE].

[34] S. He, F. Teng and Y. Zhang, String correlators: recursive expansion, integration-by-parts and scattering equations, JHEP 09 (2019) 085 [arXiv: 1907.06041] [INSPIRE].

[35] S. Stieberger, Open \& closed vs. pure open string disk amplitudes, arXiv:0907.2211 [INSPIRE].

[36] K. Zhou, Unified web for expansions of amplitudes, JHEP 10 (2019) 195 [arXiv: 1908.10272] [INSPIRE].

[37] C. Cheung, C.-H. Shen and C. Wen, Unifying relations for scattering amplitudes, JHEP 02 (2018) 095 [arXiv: 1705.03025] [INSPIRE].

[38] B. Feng, X. Li and K. Zhou, Expansion of Einstein-Yang-Mills theory by differential operators, Phys. Rev. D 100 (2019) 125012 [arXiv: 1904.05997] [INSPIRE].

[39] G. Chen, H. Johansson, F. Teng and T. Wang, On the kinematic algebra for BCJ numerators beyond the MHV sector, JHEP 11 (2019) 055 [arXiv:1906.10683] [INSPIRE]. 